\title{
Dynamic Metasurface Antennas for Uplink Massive MIMO Systems
}

\author{
Nir Shlezinger, Or Dicker, Yonina C. Eldar, Insang Yoo, Mohammadreza F. Imani, and David R. Smith
}

\begin{abstract}
Massive multiple-input multiple-output (MIMO) communications are the focus of considerable interest in recent years. While the theoretical gains of massive MIMO have been established, implementing MIMO systems with large-scale antenna arrays in practice is challenging. Among the practical challenges associated with massive MIMO systems are increased cost, power consumption, and physical size. In this work we study the implementation of massive MIMO antenna arrays using dynamic metasurface antennas (DMAs), an emerging technology which inherently handles the aforementioned challenges. Specifically, DMAs realize large-scale planar antenna arrays, and can adaptively incorporate signal processing methods such as compression and analog combining in the physical antenna structure, thus reducing the cost and power consumption. We first propose a mathematical model for massive MIMO systems with DMAs and discuss their constraints compared to ideal antenna arrays. Then, we characterize the fundamental limits of uplink communications with the resulting systems, and propose two algorithms for designing practical DMAs for approaching these limits. Our numerical results indicate that the proposed approaches result in practical massive MIMO systems whose performance is comparable to that achievable with ideal antenna arrays.
\end{abstract}

Index terms- Massive MIMO, metasurfaces, antenna design.

\section{INTRODUCTION}

Future wireless systems are required to support an increasing number of end-users with growing throughput demands. Recent years have witnessed a rising interest in massive multiple-input multiple-output (MIMO) systems, in which the base station (BS) is equipped with a large antenna array, as a method for meeting these demands and increasing the spectral efficiency (SE). In particular, it was shown that, when a sufficiently large number of antennas are utilized, the throughput can be increased in a manner which is scalable with the number of BS antennas [1].

The theoretical benefits of massive MIMO systems in terms of SE are well-established [2]-[4]. However, implementing a massive MIMO BS equipped with a standard antenna array, capable of achieving these benefits, is still a very challenging task. In particular, some of the difficulties which arise when realizing large-scale antenna arrays include high cost [5], [6], increased power consumption [7], and constrained physical

Parts of this work were accepted for presentation in the 2019 IEEE International Conference on Acoustics, Speech, and Signal Processing (ICASSP), Brighton, UK.

This project has received funding from the Air Force Office of Scientific Research under grants No. FA9550-18-1-0187 and FA9550-18-1-0208.

N. Shlezinger and Y. C. Eldar are with the faculty of Mathematics and Computer Science, Weizmann Institute of Science, Rehovot, Israel (e-mail: nirshlezinger1@gmail.com; yonina@weizmann.ac.il).

O. Dicker is with the department of Electrical Engineering, Technion, Haifa, Israel (e-mail: or.dicker@gmail.com ).

I. Yoo, M. F. Imani and D. R. Smith are with the department of Electrical and Computer Engineering, Duke University, Durham, NC (e-mail: insang.yoo@duke.edu,mohamad.imani@gmail.com; drsmith@duke.edu). size and shape [8], [9]. Several signal processing methods have been studied, aimed at tackling these difficulties. The proposed approaches include introducing analog combining to reduce the size and cost of the system [5], [10]; implementing lowresolution quantization and/or antenna selection to mitigate the power consumption [7], [11]-[15]; and utilizing efficient power amplifiers operating at reduced peak-to-average-power ratio [16], [17]. Nonetheless, all these approaches assume a fixed optimal antenna array, and attempt to tackle the difficulties which arise from this antenna array architecture from a signal processing perspective.

In parallel to the ongoing efforts to make massive MIMO feasible using signal processing techniques, a large body of research has focused on designing practical antenna arrays for massive MIMO systems [8], [9], [18], [19]. An emerging technology for realizing large-scale antenna arrays of small physical size uses metamaterial radiators instead of conventional ones. Metamaterial antennas consist of array of subwavelength metamaterial radiators, excited by a waveguide or cavity [20]. While the resulting antenna arrays typically exhibit mutual coupling and frequency selectivity, they offer comparable beam tailoring capability from a simplified hardware, which uses much less power and costs less than antenna arrays based on standard antenna arrays [21], [22]. Furthermore, a large number of tunable metamaterial antenna elements can be packed in the same physical area, [9], and metasurfaces can implement planar antennas, making it an appealing technology for supporting the increased BS deployment of $5 \mathrm{G}$ wireless networks [23, Sec. II]. Most previous works on metamaterial antennas for MIMO communications focus on designing the physical antenna structure and metamaterial substrate to satisfy desired requirements, such as gain, bandwidth, efficiency, and level of mutual correlation [9], [18], [19], [24]. Consequently, the resulting antenna structure is fixed and independent of the processing which the transmitted and received signals undergo. An alternative application for metasurfaces as reflecting elements instead of as transmit or receive antennas, was proposed in [25]-[27] as a scheme for improving energy efficiency in wireless communication networks.

Recently, dynamic metasurface antennas (DMAs) have been proposed as a method for electrically tuning the physical characteristics of metamaterial antennas [20], [28], [29]. DMAs inherently implement signal processing techniques such as beamforming, analog combining, compression, and antenna selection, without additional hardware. By introducing simple solid-state switchable components into each metamaterial element and addressing them independently, these capabilities can become reconfigurable; i.e. they can adapt to the task at hand or changes in the environment. The application of DMAs was shown to yield simple, fast, planar, and low-power systems for microwave imaging [30]-[32], radar systems [33]- 
[35], and satellite communications [36]. More recently, using cavity-backed DMAs as a novel means to generate desired patterns to enhance capacity of MIMO communications in a clustered environment has been proposed and demonstrated in numerical simulations [37]. Nonetheless, despite the potential of DMAs in combining signal processing and antenna design, their application for realizing massive MIMO systems has not yet been studied.

In this work we aim to fill this gap by studying large-scale multi-user MIMO networks utilizing DMAs. In particular, we study the achievable performance focusing on the uplink, namely, when data is transmitted from the user terminals (UTs) to the BS, and the BS is equipped with a DMA realizing a large-scale antenna array. The application of DMAs results in a simplified hardware, which inherently implements signal processing techniques such as analog combining, subject to specific constraints induced by the physics of the metasurfaces. The resulting structure can be thus used for realizing planar, compact, low cost, and spectral efficient massive MIMO BSs. Unlike standard analog combining with conventional antenna arrays, e.g., [5], [10], DMAs implement adjustable compression without requiring additional hardware.

We propose a model for MIMO systems with DMAs which encapsulates previously proposed mathematical models for the unique characteristics and constraints of these metasurfaces, such as the frequency response of each metamaterial element [20], [38], the propagation inside the waveguide [20], and the mutual coupling induced by the sub-wavelength spacing of the elements [34], [39], [40]. By integrating these established properties of DMAs into the overall MIMO system model, we obtain an equivalent communication channel including frequency selectivity and constrained linear combining, which can be analyzed using information theoretic tools. Our model also quantifies some of the gains in utilizing DMAs, demonstrating that they require less RF chains compared to standard antenna arrays, thus reducing the cost, memory requirement, and power consumption.

Next, we focus on the scenario where the wireless channel is frequency flat, and the frequency selectivity, induced by the physics of the metasurfaces, is identical among all the radiating elements. We then extend our analysis to the general scenario of frequency selective channels with an arbitrary frequency selectivity profile among the metamaterial elements. For each scenario we characterize the maximal achievable average sum-rate among all UTs in the network, and compare it to the fundamental performance limits, which is the maximal achievable sum-rate of frequency selective MIMO multiple access channels (MACs) derived in [41], and requires ideal unconstrained antenna arrays. We show that when channel is frequency flat and the frequency selectivity is identical among the elements, its effect can be accounted for in the configuration of the DMAs. Thus, under this setting, when number of DMAs is not smaller than the number of UTs, DMA based antenna arrays can approach the fundamental performance limits, achievable using ideal unconstrained antenna arrays.

For each scenario, we derive an alternating optimization algorithm for configuring the DMAs to approach the performance achievable with unconstrained antenna arrays, account- ing for the specific characteristics of the metasurfaces. Our numerical analysis demonstrates that the achievable performance of the resulting massive MIMO systems in which the BS implements its large-scale antenna array using DMAs is comparable to the theoretical fundamental limits of the channel. These limits are achievable using unconstrained antenna arrays, which are more costly, require more power and are physically larger compared to DMAs with the same number of radiators.

The rest of this paper is organized as follows: Section II introduces the mathematical formulation of DMAs, and defines the problem of uplink multi-user MIMO communications with DMAs. Section III characterizes the fundamental performance limits achievable with any antenna array, as well as the performance limits when utilizing DMA, and derives algorithms for designing DMAs to approach the optimal performance. Section IV provides simulation examples. Finally, Section V concludes the paper. Proofs of the results are detailed in the appendix.

Throughout this paper, we use boldface lower-case letters for vectors, e.g., $\mathbf{x}$; the $i$ th element of $\mathbf{x}$ is written as $(\mathbf{x})_{i}$. Matrices are denoted with boldface upper-case letters, e.g., $\boldsymbol{M}$, $(\boldsymbol{M})_{i, j}$ denotes its $(i, j)$ th element, $\operatorname{rank}(\boldsymbol{M})$ denotes its rank and $|\boldsymbol{M}|$ is its determinant. Sets are denoted with calligraphic letters, e.g., $\mathcal{X}$. We use $\boldsymbol{I}_{n}$ to denote the $n \times n$ identity matrix. Stochastic expectation and mutual information are denoted by $\mathbb{E}\{\cdot\}$ and $I(\cdot ; \cdot)$, respectively. We use $\|\cdot\|$ to denote the $\mathrm{Eu}-$ clidean norm when applied to vectors and the Frobenius norm when applied to matrices, $\otimes$ denotes the Kronecker product, $\mathcal{C}$ and $\mathcal{N}$ are the sets of complex numbers and natural numbers, respectively. For any sequence, possibly multivariate, $\mathbf{y}[i]$, and integers $b_{1}<b_{2}, \mathbf{y}_{b_{1}}^{b_{2}}$ denotes the column vector obtained by stacking $\left[\left(\mathbf{y}\left[b_{1}\right]\right)^{T}, \ldots,\left(\mathbf{y}\left[b_{2}\right]\right)^{T}\right]^{T}$ and $\mathbf{y}^{b_{2}} \equiv \mathbf{y}_{1}^{b_{2}}$.

\section{Preliminaries and Problem Formulation}

In the following we model the input-output relationship of DMAs when used on the receive side in a MIMO communications scenario. This model is based on previously proposed mathematical models for the electromagnetic properties of DMAs, in particular, on the works [20] and [34]. The main contribution of the resulting model lies in its natural integration into the overall communication system model, discussed in the following subsection, allowing the properties of DMAs to be incorporated in an equivalent channel which is analytically tractable from an information theoretic perspective, as shown in Section III. To formulate the considered setup, we first elaborate on metasurface anteannas and mathematically express the input-output relationship of DMAs in Subsection II-A. Then, we present the massive MIMO with DMAs system model in Subsection II-B. Finally, in Subsection II-C we discuss the achievable average sum-rate performance metric.

\section{A. Dynamic Metasurface Antennas}

Metamaterials are a class of artificial materials whose physical properties, and particularly their permittivity and permeability, can be engineered to exhibit a broad set of desired characteristics [42], [43]. The underlying idea behind 


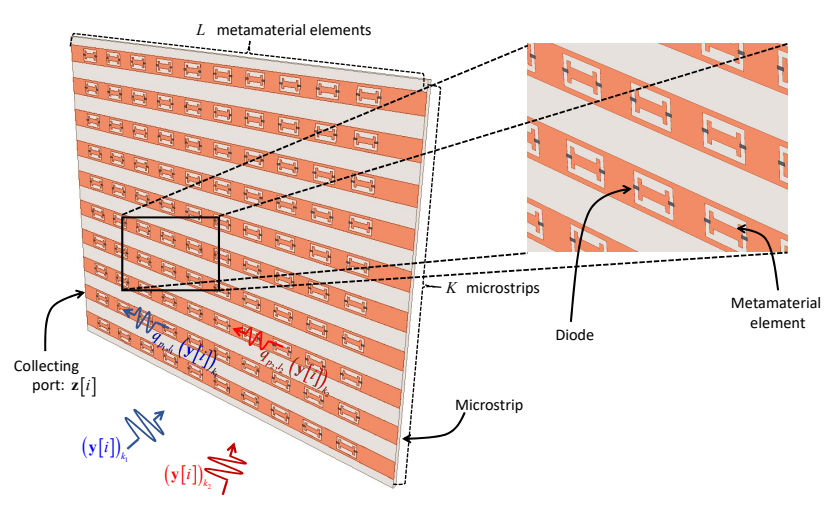

Fig. 1. Metasurface antenna illustration.

metamaterials is to introduce tailored inclusions in a host medium to emulate a desired effective property. This concept was later extended to surface configurations (thus "metasurface") where the surface effective parameters were tailored to realize a desired transformation on the transmitted, received, or reflected waves [44], [45]. More recently, metasurfaces have been implemented as radiative layers on top of a guiding structure, forming a "metasurface antenna". In a simple configuration, a metasurface antenna consists of microstrips consisting of a multitude of sub-wavelength, frequency-selective resonant metamaterial radiating elements [28]. To realize a larger antenna array, such metasurface antennas can be tiled together to form a large array. An illustration of such an array is given in Fig. 1.

On the receive side, each microstrip feeds a single RF chain, whose digital output is obtained as a linear combination of the radiation observed by each metamaterial element of the microstrip. This linear combination is a result of the following two physical phenomena:

- Frequency response of the metamaterial element: This effect can be typically modeled as a bandpass filter whose quality factor is typically around 30 [46], though higher quality factors of around 100 can also be achieved [28]. For example, at carrier frequency of $1.9 \mathrm{GHz}$, a quality factor of 30 would translate into a bandwidth of 63 $\mathrm{MHz}$. In many relevant communications scenarios, such a response can be considered as frequency flat, namely, the gain induced by the metamaterial element is the same for all the considered frequency range. It is emphasized that this does not imply that the communication channel is frequency flat, as the wireless channel gain typically varies in frequency within this band [47].

- Propagation inside the microstrip: The effect of this phenomenon depends on the location of the elements along the waveguide (e.g., the microstrip). In particular, by letting $r_{p, l}$ denote the location of the $l$ th element on the $p$ th microstrip, and $\beta_{p}$ denote the wavenumber along the waveguide, which is usually larger than the free space wavenumber $k$, the effect of the propagation inside the waveguide in the frequency domain is proportional to $e^{-j \beta_{p} \cdot r_{p, l}}$. Since the wavenumber $\beta_{p}$ is a linear function of the frequency, this effect induces non-negligible fre-

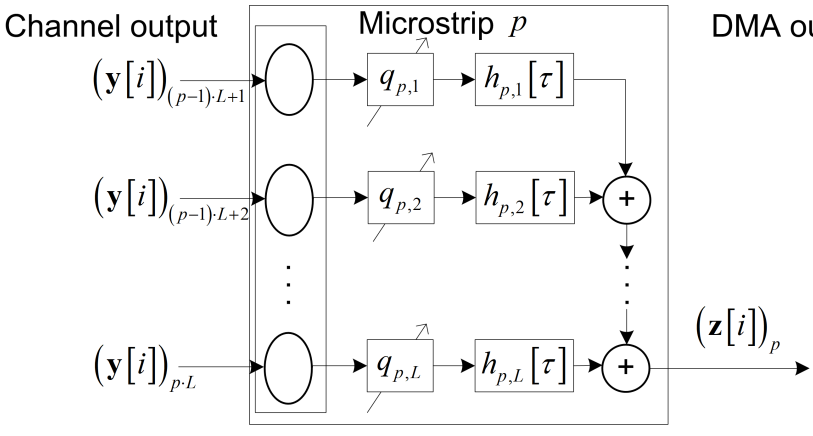

Fig. 2. Dynamic metasurface microstrip model.

quency selectivity. Thus, we henceforth model this effect in the discrete-time domain as a causal filter with finite impulse response $\left\{h_{p, l}[\tau]\right\}_{\tau=0}^{m_{h}}$ whose taps are complexvalued, i.e., $h_{p, l}[\tau] \in \mathcal{C}$, and $m_{h}$ represents the memory of the filter, namely, the number of taps.

It is worth emphasizing here that we have ignored the element-element coupling inside the microstrip for simplicity. This assumption is usually valid when metamaterial elements are weakly coupled to the guided mode [20]. For cases with strong coupling metamaterial element, one can include such coupling using coupled dipole models [39]. This model and its implications (if any) on the massive MIMO system is beyond the scope of this paper and are left for future works.

We can now mathematically formulate the input-output relationship of metasurface antennas. Consider a metasurface antenna with $K$ microstrips, each consisting of $L$ elements, and let $\mathbf{y}[i] \in \mathcal{C}^{K \cdot L \times 1}$ be a vector such that $(\mathbf{y}[i])_{(p-1) \cdot L+l}$ denotes the radiation observed at the $l$ th element of the $p$ th microstrip at time index $i$. The output of the metasurface antenna at time index $i$ is the vector $\mathbf{z}[i] \in \mathcal{C}^{K}$ whose entries can be written as

$$
(\mathbf{z}[i])_{p}=\sum_{l=1}^{L} q_{p, l} \sum_{\tau=0}^{m_{h}} h_{p, l}[\tau] \cdot(\mathbf{y}[i-\tau])_{(p-1) \cdot L+l},
$$

with $p \in\{1,2, \ldots, K\}$. An illustration of the input-output relationship induced by a single manuscript is depicted in Fig. 2. It is noted that the filters $\left\{h_{p, l}[\tau]\right\}$, representing the propagation inside each microstrip, do not depend on the gains of the metamaterial elements $\left\{q_{p, l}\right\}$, namely, we assume that the metamaterial elements do not perturb the feed wave [20]. Due to the sub-wavelength proximity of the elements in microsrtip, the input vector $\mathbf{y}[i]$ is spatially correlated, i.e., its covariance matrix is non-diagonal. We thus do not assume a specific element spacing and incorporate the resulting coupling into the general covariance of $\mathbf{y}[i]$.

The relationship between the multivariate processes $\mathbf{y}[i]$ and $\mathbf{z}[i]$ can thus be written as

$$
\mathbf{z}[i]=\boldsymbol{Q} \sum_{\tau=0}^{m_{h}} \boldsymbol{H}[\tau] \mathbf{y}[i-\tau],
$$

where $\{\boldsymbol{H}[\tau]\}_{\tau=0}^{m_{h}}$ is a set of $N \times N$ diagonal matrices, $N \triangleq$ $K \cdot L$, representing the frequency selectivity of the metasurface, i.e., $(\boldsymbol{H}[\tau])_{(p-1) L+l,(p-1) L+l}=h_{p, l}[\tau]$, and $\boldsymbol{Q}$ is an $K \times N$ 
matrix representing the configurable weights of the DMAs. Using (1), we can write

$$
(\boldsymbol{Q})_{p_{1},\left(p_{2}-1\right) K+l}=\left\{\begin{array}{ll}
q_{p_{1}, l} & p_{1}=p_{2} \\
0 & p_{1} \neq p_{2}
\end{array} .\right.
$$

For mathematical convenience, it is assumed that the coefficients $\left\{q_{p, l}\right\}$ are unitless, i.e., the polarizability of the elements is normalized. While the model detailed above considers a DMA in which the radiating elements are placed along a set of one-dimensional microstrip, it can incorporate a broader family of two-dimensional DMAs. In fact, any two-dimensional DMA in which each element is connected to a single output port can be represented via (2) by modifying the structure of the weights matrix $Q$ to represent the resulting elements interconnections.

DMAs integrate a tuning mechanism into each independent resonator of a metasurface antenna [29]. The dynamic tuning adds the flexibility to adjust the properties of the metamaterial elements, namely, to control the values of the coefficients $\left\{q_{p, l}\right\}$ in (1). The set of possible values of $\left\{q_{p, l}\right\}$, denoted $\mathcal{Q}$, represents the Lorentzian resonance response [20], and typically consists of a subset of the complex plane $\mathcal{C}$ of either of the following forms [20, Sec. III]:

- Amplitude only, namely, $\mathcal{Q}=[a, b]$ for some real nonnegative $a<b$.

- Binary amplitude, i.e., $\mathcal{Q}=c \cdot\{0,1\}$ for some fixed $c \in \mathcal{R}^{+}$.

- Lorentzian-constrained phase, that is $\mathcal{Q}=\left\{q=\frac{j+e^{j \phi}}{2}\right.$ : $\phi \in[0,2 \pi]\}$.

In order to quantify the gains of utilizing DMAs, we next compare these antenna architectures to standard antenna arrays. We use the term standard arrays for systems where the receiver is capable of directly processing the observed vector $\mathbf{y}[i]$, which is the common model in the MIMO communications literature, for both conventional MIMO [48, Ch. 7] as well as massive MIMO [2]-[4]. Clearly, any performance achievable with DMA-based antenna arrays is also achievable with standard antenna arrays, as $\mathbf{z}[i]$ can be obtained from $\mathbf{y}[i]$, but not vice versa. However, unless an additional RF chain reduction hardware is used, such as analog combiners discussed in the sequel, standard antenna arrays require each of the $K \cdot L$ radiating elements to be connected to an RF chain as well as an analog-to-digital convertor (ADC), while DMAs require a single RF chain and ADC per microstrip. Note that RF chain hardware tends to be costly [6], and that ADCs are typically a dominant source of power consumption [7] and memory usage [49]. Consequently, by utilizing DMAs, the resulting cost, memory usage, and power consumption, are reduced by a factor of $L$ compared to standard antenna arrays [37]. Additionally, DMAs can realize planar antenna arrays [21], [22], and unlike standard antenna arrays, they can pack a larger number of elements into a given physical area [9].

We note that reducing the number of RF chains and ADCs can also be carried out with standard antenna arrays using dedicated analog combining hardware, see, e.g., [5], [6], [10], [49]. However, in the presence of standard antenna arrays, analog combining comes at the cost of additional hardware, which increases the overall size and cost, especially when the analog combining should be adjustable in run-time. The exact value of the increased cost and size depends on the specific implementation of the analog combiner. DMAs inherently implement adjustable analog combining in the physical structure of the metasurfaces without additional hardware. Specifically, multiple metamaterial radiators are fed directly by a waveguide structure to simplify the feed structure, avoiding the usage of potentially more expensive and complicated RF circuitry. Thus, DMAs have been recognized as a radiative platform with a simple, energy-efficient, low-cost, and lowprofile configuration. Furthermore, standard analog combining implemented using dedicated hardware is typically subject to different constraints than those imposed on $Q$ here. In particular, while in DMAs the weights matrix $Q$ must obey the structure in (3) and its entries must be in the feasible set $\mathcal{Q}$ defined above, standard analog combiners must satisfy the architecture-based constraints detailed in [6, Sec. II], such as the commonly used phase shifting network constraint, i.e., $\mathcal{Q}=\{q \in \mathcal{C}:|q|=1\}$, or the switching network constraint, in which $\mathcal{Q}=\{0,1\}$. It is also noted that when $L=1$, $\boldsymbol{Q}=\boldsymbol{I}_{K}$, and $\left\{h_{p, l}[\tau]\right\}$ are Kronecker delta functions, namely, each microstrip realizes a single frequency flat antenna, then $\mathbf{z}[i] \equiv \mathbf{y}[i]$, and the resulting DMA coincides with the standard antenna array. However, this implementation requires the same amount of RF chains and ADCs as standard arrays, and thus does not result in any gains in terms of cost, power consumption, and memory requirement. Finally, it is emphasized that while we consider DMAs with frequency flat element response, resulting in the frequency invariant weights matrix $Q$, it is possible to design DMAs to have dynamically adjustable frequency selective weights. This can be achieved by using elements with different resonance frequency along the microstrip and turning them on and off to realize a desired frequency selective response. While designing frequency selective DMAs is expected to introduce an additional potential gain over conventional analog combiners, the set of possible frequency selectivity profiles which can be realized in DMAs is heavily implementation dependent, and as a result, we leave this for future investigation.

To summarize, DMAs realize antenna arrays with specific structure constraints, representing the underlying physics of the metasurface. These constraints include additional filtering of the received signal due to the propagation inside the microstrip; spatial correlation due to sub-wavelength element spacing; and an inherent adjustable signal compression as the signals are combined in each microstrip. The benefits of using DMAs as an antenna array architecture are their low-cost, power-efficiency, and physical shape and size flexibility. An additional benefit in the context of massive MIMO communications, which we exploit in the sequel, is their natural ability to implement a form of dynamic analog combining as an integral part of the antenna structure without requiring additional dedicated hardware.

\section{B. System Model}

We consider a noncooperative single-cell multi-user MIMO system, focusing on the uplink. The BS is equipped with a 


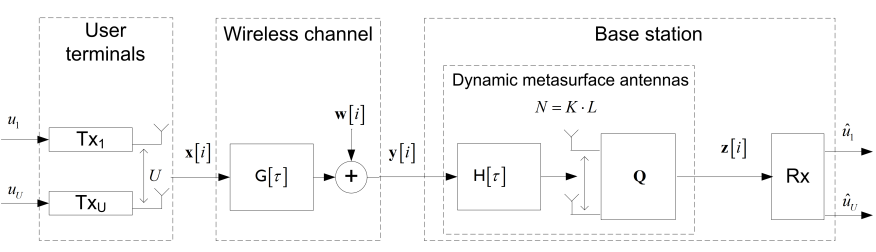

Fig. 3. System model illustration.

DMA, consisting of $K$ microstrips, each with $L$ elements, namely, the overall number of radiating elements used by the BS is $N \triangleq K \cdot L$. The number of UTs served by the BS is $U$, assumed to be not larger than $N$.

Let $\{\boldsymbol{G}[\tau]\}_{\tau=0}^{m_{g}}$ be a set of $N \times U$ matrices representing the multipath channel matrix from the UTs to the BS, where $m_{g}$ denotes the length memory of the discrete-time channel transfer function, i.e., the number of taps is $m_{g}+1$, and $m_{g}=0$ implies that the channel is memoryless. The channel output at the BS is corrupted by be an i.i.d. zero-mean proper-complex multivariate Gaussian noise $\mathbf{w}[i] \in \mathcal{C}^{N}$ with covariance matrix $C_{W}$. By letting $\mathrm{x}[i] \in \mathcal{C}^{U}$ be the transmitted signal of the UTs at time index $i$, the corresponding channel output at the BS is given by

$$
\mathbf{y}[i]=\sum_{\tau=0}^{m_{g}} \boldsymbol{G}[\tau] \mathbf{x}[i-\tau]+\mathbf{w}[i] .
$$

We assume that the UTs utilize Gaussian codebooks, i.e., $\mathbf{x}[i]$ is a zero-mean Gaussian vector with identity covariance matrix, and that the BS has full channel state information (CSI), namely, the matrices $\{\boldsymbol{G}[\tau]\}_{\tau=0}^{m_{g}}$ are known to the BS.

At the BS, the DMA converts the received signal $\mathbf{y}[i] \in \mathcal{C}^{N}$ into the vector $\mathbf{z}[i] \in \mathcal{C}^{K}$, which is used to decode the transmitted signal. As detailed in Subsection II-A, the relationship between $\mathbf{y}[i]$ and $\mathbf{z}[i]$ is given by (2). Note that the frequency selectivity of the metasurface is modeled via $\{\boldsymbol{H}[\tau]\}_{\tau=0}^{m_{h}}$. An illustration is given in Fig. 3.

We note that unlike the standard massive MIMO literature, e.g., [2], [3], which models the channel as memoryless, the model in (4) explicitly accounts for the frequency selectivity of the wireless channel. Frequency selective MIMO models as in (4), i.e., without the presence of DMAs, were studied for point-to-point communications [50], MACs [41], broadcast channels [51], and wiretap channels [52]. Our motivation for using the model in (4) stems from the fact that the standard massive MIMO memoryless model is obtained by assuming orthogonal frequency division multiplexing (OFDM) modulation with subcarrier spacing smaller than the coherence bandwidth of the channel, and cyclic prefix of length larger than the number of multipath taps [48, Ch. 3]. However, when metasurfaces are present, it is no longer reasonable to assume that the moderate frequency variations induced by the DMA are effectively canceled by the transmission scheme. Consequently, in order to account for the frequency selectivity of both the DMA as well as the wireless channel, we explicitly incorporate the effect of multipath into the model in (4).

In order to compare the performance achievable with DMA to that achievable with ideal unconstrained antenna arrays, we also consider the case where the BS decodes the transmitted signals based on the output of the wireless channel $\mathbf{y}[i]$, instead of $\mathbf{z}[i]$. This scenario is referred to henceforth as optimal MIMO. As discussed in Subsection II-A, the maximal SE of optimal MIMO is not smaller ${ }^{1}$ than that achievable with DMAs, as the output of the DMA $z[i]$ can be obtained from $\mathbf{y}[i]$. Since different configurations of the same number of elements results in a different statistical model for the channel output, in order to maintain fair comparison, the antenna spacing between the $N$ elements used in the optimal MIMO setup is identical to that used with DMAs. Under this setting, the resulting wireless channel, namely, the relationship between $\mathbf{x}[i]$ and $\mathbf{y}[i]$, is the same as in the DMA setup.

Our goal is to characterize the performance achievable for the considered system with DMAs compared to the optimal MIMO case, and to provide guidelines for configuring the DMA weights such that the performance is optimized. In the following section we properly define the performance metric used henceforth.

\section{Definitions}

In order to rigorously formulate the performance metric used in the paper, while accounting for the frequency selectivity induced by the DMAs, we present a set of necessary definitions, which are based on [53, Ch. 4]. We begin with the definition of finite-memory multi-user channels:

Definition 1 (A multi-user MIMO channel with finite-memory). A discrete-time $N \times U$ multi-user MIMO channel with finite memory consists of a set of $U$ scalar input sequences, represented via a multivariate sequence $\mathbf{x}[i] \in \mathcal{R}^{U}, i \in \mathcal{N}$, an output sequence $\mathbf{y}[i] \in \mathcal{R}^{N}, i \in \mathcal{N}$, an initial state vector $\mathbf{s}_{0} \in \mathcal{S}_{0}$ of finite dimensions, and a sequence of conditional probabilities $\left\{p\left(\mathbf{y}^{n} \mid \mathbf{x}^{n}, \mathbf{s}_{0}\right)\right\}_{n=0}^{\infty}$.

Having defined a multi-user MIMO channel with finitememory, we can now introduce the definition of codes for such channels:

Definition 2 (Multi-user code). $A\left[\left\{R_{k}\right\}_{k=1}^{U}, l\right]$ multi-user code with rates $\left\{R_{k}\right\}_{k=1}^{U}$ and blocklength $l \in \mathcal{N}$ consists of:

1) $U$ message sets $\mathcal{U}_{k} \triangleq\left\{1,2, \ldots, 2^{l R_{k}}\right\}$, with $k \in$ $\{1,2, \ldots, U\}$.

2) A family of encoders $e_{l, k}$, each maps the message $u_{k} \in$ $\mathcal{U}_{k}$ into a codeword $\mathbf{x}_{\left(u_{k}\right)}^{l}=\left[x_{\left(u_{k}\right)}[1], \ldots, x_{\left(u_{k}\right)}[l]\right]^{T}$, i.e.,

$$
e_{l, k}: \mathcal{U}_{k} \mapsto \mathcal{R}^{l} .
$$

The channel input is $\mathbf{x}[i]=\left[x_{\left(u_{1}\right)}[i], \ldots, x_{\left(u_{U}\right)}[i]\right]^{T}$.

3) A decoder $d_{l}$ which maps the channel output $\mathbf{y}^{l}$ into the messages $\hat{u}_{1}, \ldots \hat{u}_{U}$, i.e.,

$$
d_{l}: \mathcal{R}^{N \times l} \mapsto \mathcal{U}_{1} \times \cdots \times \mathcal{U}_{U} .
$$

The encoders and decoder operate independently of the initial state $\mathbf{s}_{0}$.

\footnotetext{
${ }^{1}$ To be precise, as the considered channel is information stable, the achievable sum-rate can be expressed using the mutual information between its input and output $[53$, Ch. 4]. Therefore, as $\mathbf{z}[i]$ is a deterministic mapping of $\mathbf{y}[i]$, it follows from the data processing inequality [53, Ch. 2] that the achievable sum-rate when the output $\mathbf{y}[i]$ is not smaller than that achievable when the output is $\mathbf{z}[i]$.
} 
The set $\left\{x_{\left(u_{k}\right)}^{l}\right\}_{u=1}^{2^{l R_{k}}}$ is referred to as the $k$-th codebook of the $\left[\left\{R_{k}\right\}_{k=1}^{U^{k}}, l\right]$ code. Assuming each message $u_{k}$ is uniformly selected from $\mathcal{U}_{k}$, the average probability of error, when the initial state is $\mathbf{s}_{0}^{\prime}$, is given by [51, Sec. III]:

$$
\begin{aligned}
P_{\mathrm{e}}^{l}\left(\mathbf{s}_{0}^{\prime}\right)=\frac{1}{2^{l} \sum_{k=1}^{U} R_{k}} \sum_{u_{1}=1}^{2^{l R_{1}}} \cdots \sum_{u_{U}=1}^{2^{l R_{U}}} \operatorname{Pr}\left(d_{l}\left(\mathbf{y}^{l}\right) \neq\left\{u_{i}^{\prime}\right\}\right. \\
\left.\qquad\left\{u_{i}=u_{i}^{\prime}\right\}, \mathbf{s}_{0}=\mathbf{s}_{0}^{\prime}\right) .
\end{aligned}
$$

Using Definition 2, we can now properly formulate the definition for achievable sum-rate, which will be used henceforth as our main metric for evaluating multi-user MIMO networks operating with DMAs.

Definition 3 (Achievable average sum-rate). An average sumrate $R_{\mathrm{S}}$ is called achievable if, for every $\epsilon_{1}, \epsilon_{2}>0$, there exists a positive integer $l_{0}>0$ such that for all integer $l>l_{0}$, there exists a multi-user code, $\left[\left\{R_{k}\right\}_{k=1}^{U}, l\right]$, which satisfies

$$
\sup _{\mathbf{s}_{0}^{\prime} \in \mathcal{S}_{0}} P_{\mathrm{e}}^{l}\left(\mathbf{s}_{0}^{\prime}\right)<\epsilon_{1},
$$

and

$$
\frac{1}{U} \sum_{k=1}^{U} R_{k} \geq R_{\mathrm{s}}-\epsilon_{2} .
$$

Note that the achievable average sum-rate in Definition 3 is a fundamental property of the multi-user MIMO channel. In fact, for a given set of multi-user encoders, the decoder which maximizes the achievable average sum-rate typically implements joint decoding [53, Ch. 4]. Such decoders are usually computationally complex, and thus many works on massive MIMO focus on the achievable average sum-rate assuming less complex suboptimal separate linear decoding, see [2]-[4]. In this work we focus on the implementation of the antenna array using DMAs, with the promise of reducing cost, size, and power consumption. Consequently, we impose no constraints on the processing and decoding carried out in the digital domain, assume that the BS has perfect knowledge of the underlying channel, and characterize the performance in terms of the maximal achievable average sum-rate. It is emphasized that operating under computational complexity constraints and obtaining an accurate channel estimation are challenging tasks on their own for massive MIMO BSs with DMAs. We consider the design of efficient decoding and channel estimation schemes, as well the analysis of the effect of inaccurate channel knowledge and hardware impairments on the resulting performance when using DMAs, as potential future research directions, extending the current study.

\section{Achievable Average Sum-Rates}

In the following we study the achievable average sum-rate and the resulting DMA configuration for the setup presented in Subsection II-B. To formulate the achievable average sumrate, let $\boldsymbol{\Gamma}(\omega)$ and $\boldsymbol{\Sigma}(\omega), \omega \in[0,2 \pi)$, denote the discrete-time Fourier transforms (DTFTs) of $\boldsymbol{H}[\tau]$ and of $\boldsymbol{G}[\tau]$, respectively. The maximal achievable average sum-rate for a fixed DMA weights matrix $Q$ is stated in the following theorem:
Theorem 1. The maximal achievable average sum-rate of the channel in (4) and (2) for a fixed weight matrix $\boldsymbol{Q}$ is given by

$$
\begin{aligned}
R_{\mathrm{s}}=\frac{1}{2 \pi \cdot U} \int_{0}^{2 \pi} \log \mid & \boldsymbol{I}_{K}+\boldsymbol{Q} \boldsymbol{\Gamma}(\omega) \boldsymbol{\Sigma}(\omega) \boldsymbol{\Sigma}^{H}(\omega) \boldsymbol{\Gamma}^{H}(\omega) \boldsymbol{Q}^{H} \\
& \times\left(\boldsymbol{Q} \boldsymbol{\Gamma}(\omega) \boldsymbol{C}_{W} \boldsymbol{\Gamma}^{H}(\omega) \boldsymbol{Q}^{H}\right)^{-1} \mid d \omega .
\end{aligned}
$$

Proof: See Appendix A.

Theorem 1 characterizes the maximal achievable sum-rate by incorporating the DMA operation as part of the channel, and obtaining the achievable sum-rate of the resulting finitememory MAC as in [41]. Theorem 1 can also be used to obtain the fundamental performance limits of the wireless channel, achievable with optimal unconstrained antenna arrays, as stated in the following corollary:

Corollary 1. Define $\tilde{\boldsymbol{\Sigma}}(\omega) \triangleq \boldsymbol{C}_{W}^{-1 / 2} \boldsymbol{\Sigma}(\omega) \boldsymbol{\Sigma}^{H}(\omega) \boldsymbol{C}_{W}^{-1 / 2}$, and let $\left\{\lambda_{i}(\omega)\right\}_{i=1}^{U}$ be its eigenvalues arranged in descending order. The maximal achievable average sum-rate of the optimal MIMO setup is given by

$$
R_{\mathrm{s}}^{\mathrm{OM}}=\frac{1}{2 \pi \cdot U} \int_{0}^{2 \pi} \sum_{i=1}^{U} \log \left(1+\lambda_{i}(\omega)\right) d \omega .
$$

Proof: As noted in Subsection II-A, when $L=1, \boldsymbol{Q}=$ $\boldsymbol{I}_{K}$, and $\boldsymbol{\Gamma}(\omega) \equiv \boldsymbol{I}_{K}$, the resulting setup coincides with the optimal MIMO setup. Substituting this into (7) proves (8).

When DMAs are utilized, we note that due to the integration operation and the structure constraints on $Q$, it is difficult to determine the DMA weights matrix $Q$ such that (7) is maximized. Therefore, in order to design $Q$ and obtain the resulting $R_{\mathrm{s}}$, we first focus on the special case where all the metasurface elements exhibit the same frequency selectivity profile, and the wireless channel is frequency flat. For this case, we derive in Subsection III-A the choice of $\boldsymbol{Q}$ which maximizes the achievable sum-rate, ignoring the structure constraints detailed in Subsection II-A. Then, in Subsection III-B we propose an iterative algorithm for configuring practical constrained DMAs. Finally, in Subsection III-C, we show how these design principles can be extended to arbitrary frequency selectivity profiles.

\section{A. Optimal Weights for Flat Channels with Identical Fre- quency Selectivity}

The maximal achievable average sum-rate and the corresponding weights configuration $\boldsymbol{Q}$ which maximizes (7) are in general difficult to compute. Thus, we will first consider the special case where all the metamaterial elements exhibit the same frequency selectivity profile, and the wireless channel is frequency flat. Under this model, the multivariate filter representing the response of the antennas $\boldsymbol{H}[\tau]$ can be written as $\boldsymbol{H}[\tau]=\boldsymbol{I}_{n_{t}} \cdot h[\tau]$, for some scalar mapping $h[\tau]$, and the multipath channel is given by a single tap $\boldsymbol{G}=\boldsymbol{G}[0]$, i.e., $m_{g}=0$. By letting $\gamma(\omega)$ be the DTFT of $h[\tau]$, the multivariate DTFTs of $\boldsymbol{H}[\tau]$ and of $\boldsymbol{G}[\tau]$ can be written $\boldsymbol{\Gamma}(\omega)=\boldsymbol{I}_{n_{t}} \cdot \gamma(\omega)$ 
and $\boldsymbol{\Sigma}(\omega)=\boldsymbol{G}$. Thus, the achievable average sum-rate in (7) is given by

$$
R_{\mathrm{s}}=\frac{1}{U} \log \left|\boldsymbol{I}_{K}+\boldsymbol{Q} \boldsymbol{G} \boldsymbol{G}^{H} \boldsymbol{Q}^{H}\left(\boldsymbol{Q} \boldsymbol{C}_{W} \boldsymbol{Q}^{H}\right)^{-1}\right| .
$$

In order to find $\boldsymbol{Q}$ which maximizes (9), we define $\tilde{\boldsymbol{G}} \triangleq$ $\boldsymbol{C}_{W}^{-1 / 2} \boldsymbol{G} \boldsymbol{G}^{H} \boldsymbol{C}_{W}^{-1 / 2}$. We now formulate the dependence of $R_{\mathrm{s}}$ on $Q$ in the following lemma:

Lemma 1. Define $\tilde{\boldsymbol{Q}} \triangleq \boldsymbol{Q} C_{W}^{1 / 2}$ and let $\boldsymbol{V}$ be its right singular vectors matrix. By letting $\tilde{\boldsymbol{V}}$ be the $N \times K$ matrix consisting of the first $K$ columns of the unitary matrix $\boldsymbol{V}$, the achievable sum-rate in (9) can be written as

$$
R_{\mathrm{s}}=\frac{1}{U} \log \left|\boldsymbol{I}_{K}+\tilde{\boldsymbol{V}}^{H} \tilde{\boldsymbol{G}} \tilde{\boldsymbol{V}}\right| .
$$

Proof: By replacing $\boldsymbol{Q}$ in (9) with $\tilde{\boldsymbol{Q}}=\boldsymbol{Q} \boldsymbol{C}_{W}^{1 / 2}$ it follows from Sylvester's determinant theorem [54, Ch. 6.2] that

$$
R_{\mathrm{s}}=\frac{1}{U} \log \left|\boldsymbol{I}_{N}+\tilde{\boldsymbol{G}}\left(\tilde{\boldsymbol{Q}}^{H}\left(\tilde{\boldsymbol{Q}} \tilde{\boldsymbol{Q}}^{H}\right)^{-1} \tilde{\boldsymbol{Q}}\right)\right|
$$

Next, we note that $\tilde{Q}^{H}\left(\tilde{Q} \tilde{Q}^{H}\right)^{-1} \tilde{\boldsymbol{Q}}$ is a projection matrix, and can therefore be written as $\tilde{\boldsymbol{Q}}^{H}\left(\tilde{\boldsymbol{Q}} \tilde{\boldsymbol{Q}}^{H}\right)^{-1} \tilde{\boldsymbol{Q}}=\tilde{\boldsymbol{V}} \tilde{\boldsymbol{V}}^{H}$ [54, Ch. 5.9]. Substituting this into (11) proves (10).

Lemma 1 implies that the achievable average sum-rate $R_{\mathrm{S}}$ depends on the weights matrix $Q$ only through the first $K$ right eigenvectors of $\tilde{\boldsymbol{Q}}=Q C_{W}^{1 / 2}$. If we ignore the structure constraints on $Q$, then the maximal achievable sum-rate and the corresponding choice of $\tilde{\boldsymbol{V}}$ which maximizes (11) are given in the following corollary:

Corollary 2. Let $\left\{\lambda_{i}\right\}_{i=1}^{U}$ be the eigenvalues of $\tilde{\boldsymbol{G}}$ arranged in descending order. Then, the maximal achievable average sum-rate when $Q$ can be any complex matrix is given by

$$
R_{\mathrm{s}}^{\mathrm{OD}}=\frac{1}{U} \sum_{i=1}^{\min (K, U)} \log \left(1+\lambda_{i}\right)
$$

The rate $R_{\mathrm{S}}^{\mathrm{OD}}$ achieved by setting the columns of $\tilde{\boldsymbol{V}}$ to be the eigenvectors corresponding to $\left\{\lambda_{i}\right\}_{i=1}^{U}$.

Proof: The corollary follows directly from (11).

Note that the number of non-zero eigenvalues of $\tilde{\boldsymbol{G}}$ is equal to its $\operatorname{rank}, \operatorname{denoted} \operatorname{rank}(\tilde{\boldsymbol{G}})$, which is at most $U$. It therefore follows from (12) that increasing the number of microstrips $K$ to be larger than $\operatorname{rank}(\tilde{\boldsymbol{G}})$ has no effect on the optimal sum-rate $R_{\mathrm{S}}^{\mathrm{OD}}$. In particular, comparing (12) to the fundamental limits in (8), we note that when $K \geq \operatorname{rank}(\tilde{\boldsymbol{G}})$, then $R_{\mathrm{S}}^{\mathrm{OD}}$ achieves the fundamental limits $R_{\mathrm{s}}^{\mathrm{OM}}$. However, as each microstrip requires a single RF chain and ADC, increasing $K$ implicitly increases the cost, power usage, and memory requirements of the resulting system. Furthermore, by letting $\tilde{\boldsymbol{U}} \tilde{\boldsymbol{D}} \tilde{\boldsymbol{V}}^{H}$ be the compact singular valued decomposition (SVD) of the optimal $\tilde{Q}$, it follows from Corollary 2 that the weights matrix which maximizes (9) can be written as

$$
\boldsymbol{Q}^{\mathrm{OD}}=\tilde{\boldsymbol{U}} \tilde{\boldsymbol{D}} \tilde{\boldsymbol{V}}^{H} \boldsymbol{C}_{W}^{-1 / 2}
$$

In particular, the matrix in (13) maximizes (9) for any setting of unitary $K \times K$ matrix $\tilde{\boldsymbol{U}}$ and diagonal $K \times K$ matrix $\tilde{\boldsymbol{D}}$ with positive diagonal entries.

It follows from (13) that the optimal weights matrix $Q^{\mathrm{OD}}$ implements the following processing: First, it applies a noise whitening filter, modeled via the matrix $C_{W}^{-1 / 2}$. Then, it utilizes the transformation $\tilde{\boldsymbol{V}}^{H}$ to project the output into its least noisy $K \times 1$ subspace, determined by the largest singular values of the whitened channel transfer matrix $\boldsymbol{C}_{W}^{-1 / 2} \boldsymbol{G}$, or alternatively, by the largest eigenvalues of $\tilde{\boldsymbol{G}}$. The fact that $Q^{\mathrm{OD}}$ depends on the channel and the statistics of the noise indicates that the ability to reconfigure the analog combining weights, which is inherently supported by DMAs, is vital in wireless communications. Finally, we note that the remaining invertible processing, determined by the matrices $\tilde{U}, \tilde{D}$, has no effect on the resulting achievable rate, in agreement with the data processing inequality [53, Ch. 2.3]. However, in the following subsection we show that these matrices can be used to facilitate the approximation of $Q^{\mathrm{OD}}$ via a feasible weights matrix, which satisfies (3) and whose entries belong to $\mathcal{Q}$.

\section{B. Practical Design for Flat Channels with Identical Fre-} quency Selectivity

The derivation of the optimal sum-rate in Corollary 2 ignores the structure constraints on $Q$, and assumes that the right eigenvectors matrix $\tilde{\boldsymbol{V}}$ can be any set of unitary vectors. Nonetheless, as detailed in the problem formulation in Subsection II-B, $\boldsymbol{Q}$ must be written as in (3), and its coefficients $\left\{q_{i, l}\right\}$ should belong to the feasible set $\mathcal{Q}$. Since finding the constrained matrix $Q$ which maximizes (9) is a difficult task, we propose to set $Q$ to be the closest feasible matrix to the unconstrained $Q^{\mathrm{OD}}$ in the sense of minimal Frobenious norm. Here, as in [10], [55], we exploit the fact that $R_{\mathrm{s}}^{\mathrm{OD}}$ is invariant to the selection of the left singular matrix $\tilde{U}$ and the diagonal singular values matrix $\tilde{D}$, and set these matrices such that the Frobenious distance to the feasible approximation is minimized. To formulate the problem, we let $\mathcal{Q}^{K \times N}$ be the set of $K \times N$ which can be written as in (3) and whose non-zero entries belong to the feasible set $\mathcal{Q}$. Let $\mathcal{U}^{K}$ denote the set of $K \times K$ unitary matrices, and $\mathcal{D}^{K}$ be the set of $K \times K$ diagonal matrices with positive diagonal entries. Specifically, we fix some $\epsilon>0$ and restrict the diagonal entires of the matrices in $\mathcal{D}^{K}$ to be not smaller than $\epsilon$. We set the weights matrix $Q$ to be the solution to:

$$
\min _{\boldsymbol{Q} \in \mathcal{Q}^{K \times N}, \tilde{\boldsymbol{U}} \in \mathcal{U}^{K}, \tilde{\boldsymbol{D}} \in \mathcal{D}^{K}}\left\|\boldsymbol{Q}-\tilde{\boldsymbol{U}} \tilde{\boldsymbol{D}} \tilde{\boldsymbol{V}}^{H} \boldsymbol{C}_{W}^{-1 / 2}\right\|^{2} .
$$

Let $P_{\mathcal{Q}}: \mathcal{C}^{K \times N} \mapsto \mathcal{Q}^{K \times N}$ be the entry-wise projection into $\mathcal{Q}^{K \times N}$. By (3) the entry-wise projection of $M \in \mathcal{C}^{K \times N}$ is given by

$$
\begin{aligned}
& \left(P_{\mathcal{Q}}(\boldsymbol{M})\right)_{p_{1},\left(p_{2}-1\right) K+l}= \\
& \begin{cases}\underset{q \in \mathcal{Q}}{\arg \min }\left|q-(\boldsymbol{M})_{p_{1},\left(p_{2}-1\right) K+l}\right|^{2} & p_{1}=p_{2} \\
0 & p_{1} \neq p_{2} .\end{cases}
\end{aligned}
$$

In order to solve (14), we propose an alternating minimization algorithm, based on the properties detailed in the following lemma: 
Lemma 2. For any $M \in \mathcal{C}^{K \times N}$ we have that

$$
\boldsymbol{Q}^{\mathrm{AM}}(\boldsymbol{M}) \triangleq \underset{\boldsymbol{Q} \in \mathcal{Q}^{K \times N}}{\arg \min }\|\boldsymbol{Q}-\boldsymbol{M}\|^{2}=P_{\mathcal{Q}}(\boldsymbol{M}) .
$$

Additionally, for any $\boldsymbol{M}_{1}, \boldsymbol{M}_{2} \in \mathcal{C}^{K \times N}$, let $\boldsymbol{U}_{M}$ and $\boldsymbol{V}_{M}$ be the left singular vectors matrix and the right singular vectors matrix of $M_{1} M_{2}^{H}$, then

$$
\begin{aligned}
\tilde{\boldsymbol{U}}^{\mathrm{AM}}\left(\boldsymbol{M}_{1}, \boldsymbol{M}_{2}\right) & \triangleq \underset{\tilde{\boldsymbol{U}} \in \mathcal{U}^{K}}{\arg \min }\left\|\boldsymbol{M}_{1}-\tilde{\boldsymbol{U}} \boldsymbol{M}_{2}\right\|^{2} \\
& =\boldsymbol{U}_{M} \boldsymbol{V}_{M}^{H} .
\end{aligned}
$$

Finally, by letting $\mathbf{m}_{1, i}$ and $\mathbf{m}_{2, i}$ be the ith columns of $\boldsymbol{M}_{1}^{H}$ and $M_{2}^{H}$, respectively, $i \in\{1,2, \ldots, K\}$, we have that the diagonal entries of

$$
\tilde{\boldsymbol{D}}^{\mathrm{AM}}\left(\boldsymbol{M}_{1}, \boldsymbol{M}_{2}\right) \triangleq \underset{\tilde{\boldsymbol{D}} \in \mathcal{D}^{K}}{\arg \min }\left\|\boldsymbol{M}_{1}-\tilde{\boldsymbol{D}} \boldsymbol{M}_{2}\right\|^{2},
$$

are given by

$$
\left(\tilde{D}^{\mathrm{AM}}\left(\boldsymbol{M}_{1}, \boldsymbol{M}_{2}\right)\right)_{i, i}=\max \left(\frac{\operatorname{Re}\left(\mathbf{m}_{1, i}^{H} \mathbf{m}_{2, i}\right)}{\left\|\mathbf{m}_{2, i}\right\|^{2}}, \epsilon\right) .
$$

Proof: See Appendix B.

Based on Lemma 2, we propose to solve the joint optimization problem (14) in an alternating fashion, i.e., optimize over $Q$ for fixed $\tilde{U}, \tilde{D}$, next optimize over $\tilde{U}$ for fixed $Q, \tilde{D}$, then optimize over $\tilde{D}$ for fixed $\boldsymbol{Q}, \tilde{\boldsymbol{U}}$, and continue until convergence. The resulting alternating minimization algorithm is summarized in Algorithm 1. As the Frobenious norm objective in (14) is differentiable, convergence of the alternating optimization algorithm is guaranteed [57, Thm. 2].

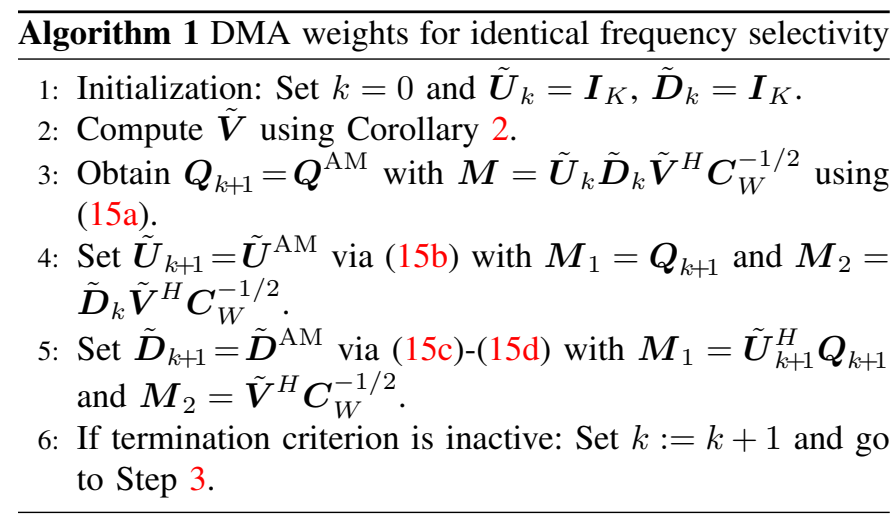

In Algorithm 1 we exploit the fact that the optimal unconstrained $Q^{\mathrm{OD}}$ achieves the same sum-rate for any setting of $\tilde{\boldsymbol{U}}, \tilde{D}$, and use these matrices as optimization variables. Consequently, we are able to obtain feasible weight matrices which are within a small distance from the optimal unconstrained matrix. In our numerical study in Section IV we demonstrate that BSs equipped with DMAs designed via Algorithm 1 are capable of achieving performance which is within a small gap of the fundamental limits of the channel, achievable using optimal antenna arrays. Furthermore, it is illustrated that, unlike the unconstrained case, when $\boldsymbol{Q} \in \mathcal{Q}^{K \times N}$, increasing the number of microstrips $K$ above $U$ increases the achievable sum-rate, as the resulting $Q^{\mathrm{OD}}$ can be better approximated using a feasible matrix.

\section{Practical Design for Arbitrary Frequency Selectivity}

In the previous subsections we studied the special case where the wireless channel is frequency flat and the metamaterial elements exhibit the same frequency selectivity profile, i.e., $m_{g}=0$ and $\boldsymbol{H}[\tau]=\boldsymbol{I}_{n_{t}} \cdot h[\tau]$, for some scalar mapping $h[\tau]$. Under this setting, we were able to express the integral in (7) with a single log-det expression in (9), as the wireless channel is memoryless and the effect of $h[\tau]$ on the transmitted signal was canceled by its contribution to the effective noise. However, wireless channels are typically frequency selective, and in practical metasurfaces, each element may exhibit a different frequency selectivity profile. Here, the frequency selectivity cannot be effectively canceled, and the explicit value of $\boldsymbol{H}[\tau]$ has to be accounted for. In the following we show how the design principles proposed in the previous subsections can be extended to this general case.

To that aim, we fix some positive integer $B$, and define $\omega_{i} \triangleq \frac{2 \pi \cdot i}{B}, i \in\{1,2, \ldots, B\}$. We can now approximate (7) as

$$
\begin{array}{r}
R_{\mathrm{s}} \approx \frac{1}{B \cdot U} \sum_{i=1}^{B} \log \mid \boldsymbol{I}_{K}+\boldsymbol{Q} \boldsymbol{\Gamma}\left(\omega_{i}\right) \boldsymbol{\Sigma}\left(\omega_{i}\right) \boldsymbol{\Sigma}^{H}\left(\omega_{i}\right) \boldsymbol{\Gamma}^{H}\left(\omega_{i}\right) \boldsymbol{Q}^{H} \\
\left(\boldsymbol{Q} \boldsymbol{\Gamma}\left(\omega_{i}\right) \boldsymbol{C}_{W} \boldsymbol{\Gamma}^{H}\left(\omega_{i}\right) \boldsymbol{Q}^{H}\right)^{-1} \mid
\end{array}
$$

Note that as $B$ increases, (16) approaches the actual sumrate in (7). We next write (16) in terms of a single log-det expression, as in (9). To that aim, let $\operatorname{BlkDiag}\left(\left\{\boldsymbol{A}_{i}\right\}_{i=1}^{B}\right)$ be a block diagonal matrix with diagonal submatrices $\left\{\boldsymbol{A}_{i}\right\}_{i=1}^{B}$, and define the $B \cdot N \times B \cdot N$ block diagonal matrices $\overline{\boldsymbol{G}} \triangleq \operatorname{BlkDiag}\left(\left\{\boldsymbol{\Gamma}\left(\omega_{i}\right) \boldsymbol{\Sigma}\left(\omega_{i}\right)\right\}_{i=1}^{B}\right)$ and $\overline{\boldsymbol{C}}_{W} \triangleq$ $\operatorname{BlkDiag}\left(\left\{\boldsymbol{\Gamma}\left(\omega_{i}\right) \boldsymbol{C}_{W} \boldsymbol{\Gamma}^{H}\left(\omega_{i}\right)\right\}_{i=1}^{B}\right)$. Also, define $\overline{\boldsymbol{Q}} \triangleq \boldsymbol{I}_{B} \otimes$ $\boldsymbol{Q}$. Using these notations, it follows from [54, Pg. 122] that

$$
\begin{aligned}
\boldsymbol{I}_{B \cdot K}+\overline{\boldsymbol{Q}} \overline{\boldsymbol{G}} \overline{\boldsymbol{G}}^{H} & \overline{\boldsymbol{Q}}^{H}\left(\overline{\boldsymbol{Q}} \overline{\boldsymbol{C}}_{W} \overline{\boldsymbol{Q}}^{H}\right)^{-1} \\
=\operatorname{BlkDiag}( & \left\{\boldsymbol{I}_{K}+\boldsymbol{Q} \boldsymbol{\Gamma}\left(\omega_{i}\right) \boldsymbol{\Sigma}\left(\omega_{i}\right) \boldsymbol{\Sigma}^{H}\left(\omega_{i}\right) \boldsymbol{\Gamma}^{H}\left(\omega_{i}\right) \boldsymbol{Q}^{H}\right. \\
& \left.\left.\times\left(\boldsymbol{Q} \boldsymbol{\Gamma}\left(\omega_{i}\right) \boldsymbol{C}_{W} \boldsymbol{\Gamma}^{H}\left(\omega_{i}\right) \boldsymbol{Q}^{H}\right)^{-1}\right\}_{i=1}^{B}\right) .
\end{aligned}
$$

Since $\left|\operatorname{BlkDiag}\left(\left\{\boldsymbol{A}_{i}\right\}_{i=1}^{B}\right)\right|=\prod_{i=1}^{B}\left|\boldsymbol{A}_{i}\right|$ when $\boldsymbol{A}_{i}$ are square matrices [54, Pg. 467], it follows that (16) can be written as

$$
R_{\mathrm{s}} \approx \frac{1}{B \cdot U} \log \left|\boldsymbol{I}_{B \cdot K}+\overline{\boldsymbol{Q}} \overline{\boldsymbol{G}} \overline{\boldsymbol{G}}^{H} \overline{\boldsymbol{Q}}^{H}\left(\overline{\boldsymbol{Q}} \overline{\boldsymbol{C}}_{W} \overline{\boldsymbol{Q}}^{H}\right)^{-1}\right| .
$$

The approximation in (17) implies that the expression for the achievable sum-rate with arbitrary frequency selectivity is similar to that with identical frequency selectivity and flat channels in (9). Consequently, the design principles used for configuring the DMA to minimize (9) in Algorithm 1 can also be used to minimize (17). The main difference between minimizing (9) and (17) is that in (17), the equivalent weights matrix $\overline{\boldsymbol{Q}}$ has to be written as $\boldsymbol{I}_{B} \otimes \boldsymbol{Q}$ where $\boldsymbol{Q} \in \mathcal{Q}^{K \times N}$. This additional constraint can be accounted for in the alternating minimization algorithm using the following lemma: 
Lemma 3. For any $\boldsymbol{M} \in \mathcal{C}^{B \cdot K \times B \cdot N}$, the weights matrix which minimizes

$$
\boldsymbol{Q}^{\mathrm{AM} 2}(\boldsymbol{M}) \triangleq \underset{\boldsymbol{Q} \in \mathcal{Q}^{K \times N}}{\arg \min }\left\|\left(\boldsymbol{I}_{B} \otimes \boldsymbol{Q}\right)-\boldsymbol{M}\right\|^{2},
$$

is given by

$$
\begin{aligned}
& \left(\boldsymbol{Q}^{\mathrm{AM} 2}(\boldsymbol{M})\right)_{p_{1},\left(p_{2}-1\right) K+l}= \\
& \begin{cases}\underset{q \in \mathcal{Q}}{\arg \min } \sum_{i=0}^{B-1}\left|q-(\boldsymbol{M})_{i \cdot K+p_{1}, i \cdot N+\left(p_{2}-1\right) K+l}\right|^{2} & p_{1}=p_{2} \\
0 & p_{1} \neq p_{2} .\end{cases}
\end{aligned}
$$

Proof: The lemma is obtained by explicitly writing the Frobenious norm in (18), noting that each element of $Q$ independently effects the overall norm via the sum of $B$ terms, as given in the lemma.

Note that for $\mathcal{Q}=\mathcal{C}$, the non-zero entries of $Q^{\mathrm{AM} 2}$ are given by the sample mean of their corresponding entries in $\boldsymbol{M}$. Using Lemma 3, we can now adapt Algorithm 1 to account for arbitrary frequency selectivity profiles, resulting in Algorithm 2.

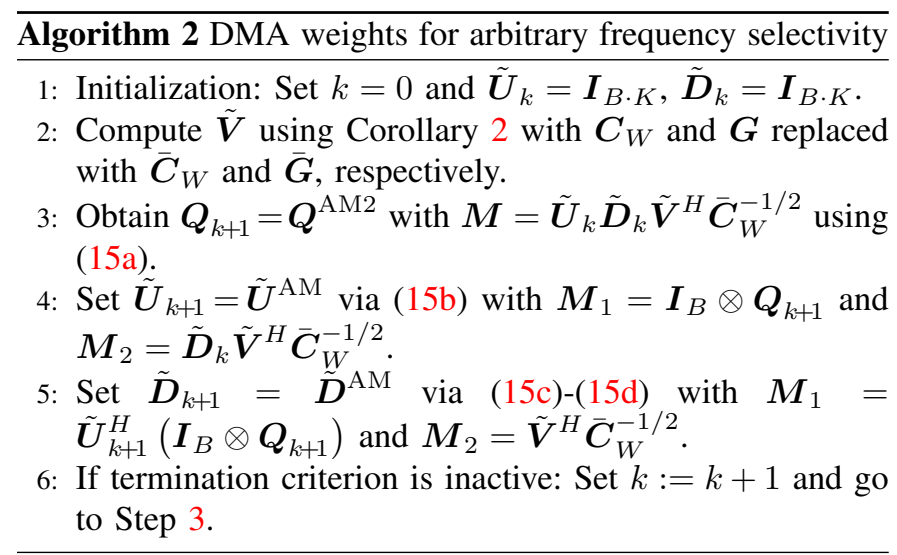

In order to evaluate the gap of the resulting configuration from optimality, we wish to characterize the maximal sum-rate achievable when $\boldsymbol{Q}$ can be any matrix in $\mathcal{C}^{K \times N}$, not restricted to satisfy (3). Since $Q$ in (7) does not vary with $\omega$, obtaining the optimal performance is a difficult task. Nonetheless, (7) can be used to obtain an upper bound on the optimal average sum-rate, as stated in the following proposition:

Proposition 1. If $\boldsymbol{\Gamma}(\omega)$ is non-singular for every $\omega \in[0,2 \pi)$, then the maximal achievable average sum-rate is upperbounded by

$$
R_{\mathrm{s}} \leq \frac{1}{2 \pi \cdot U} \int_{0}^{2 \pi} \sum_{i=1}^{\min (U, K)} \log \left(1+\lambda_{i}(\omega)\right) d \omega,
$$

where $\left\{\lambda_{i}(\omega)\right\}_{i=1}^{U}$ are defined in Corollary 1 .

Proof: See Appendix C.

It is emphasized that the upper bound in (19) is in general very difficult to approach in practice, as it is computed by allowing the DMA weights to be frequency selective, thus effectively canceling the frequency selectivity of the wireless channel and the different elements in the microstrips. As in our design we assume that the DMA weights do not vary in frequency, the resulting system in general cannot achieve the bound in Proposition 1. Nonetheless, in the numerical evaluations in Section IV it is demonstrated that BSs utilizing practical DMAs designed via Algorithm 2 are capable of achieving performance which is comparable with the upper bound in (19). In particular, we show that, when using properly configured DMAs, the resulting achievable average sum-rate is within a reasonable gap from the upper bound and that both curves scale similarly with respect to signal-to-noise ratio (SNR)

By repeating the arguments in the discussion following Corollary 2, it holds that when $K$ is not smaller than the rank of $\tilde{\Sigma}(\omega)$ for each $\omega \in[0,2 \pi]$, then the upper bound in (19) coincides with the fundamental performance limits $R_{\mathrm{s}}^{\mathrm{OM}}$ given in Corollary 1.

\section{NUMERICAL STUdY}

In this section we numerically evaluate the achievable performance using the DMA configurations derived in Section III. First, in Subsection IV-A we consider frequency flat channels with DMAs in which each element exhibits the same frequency selectivity profile, and numerically evaluate the average sum-rate achievable using the DMA design in Algorithm 1. Then, in Subsection IV-B, we study frequency selective channels with DMAs in which each element exhibits a different frequency selectivity, and compute the achievable performance of the DMA configuration obtained using Algorithm 2.

We consider an uplink multi-user MIMO cell in a rich scattering environment. In this setup, a BS equipped with a DMA serves $U=10$ UTs, uniformly distributed in a hexagonal cell of radius $400 \mathrm{~m}$, with the exception of a circle with radius $20 \mathrm{~m}$ around the BS. An illustration of such a system is depicted in Fig. 4. We use $\rho_{i}$ to denote the distance of the $i$ th UT from the BS. Based on the model for frequency selective wireless MIMO channel proposed in [58], the channel transfer matrices $\{\boldsymbol{G}[\tau]\}_{\tau=0}^{m_{g}}$ are generated as $\boldsymbol{G}[\tau]=\sigma_{\boldsymbol{G}}^{2}[\tau] \boldsymbol{\Sigma}_{R}^{1 / 2} \boldsymbol{G}_{R}[\tau] \boldsymbol{D}[\tau]$, where:

- $\left\{\sigma_{G}^{2}[\tau]\right\}_{\tau=0}^{m_{g}}$ is the relative path loss of each tap, given by an exponentially decaying profile, i.e., $\sigma_{G}^{2}[\tau]=e^{-\tau}$.

- $\left\{\boldsymbol{G}_{R}[\tau]\right\}_{\tau=0}^{m_{g}}$ are a set of i.i.d. proper-complex zeromean Gaussian $N \times U$ matrices with i.i.d. entires of unit variance.

- $\boldsymbol{\Sigma}_{R}$ is an $N \times N$ representing the correlation induced by the sub-wavelength spacing of the elements in each microstrip. Neglecting the coupling between different microstrips, we set $\boldsymbol{\Sigma}_{R}=\boldsymbol{I}_{K} \otimes \boldsymbol{\Sigma}_{M}$, where $\boldsymbol{\Sigma}_{M} \in \mathcal{C}^{L \times L}$ models the coupling induced between the elements of the same microstrip. In particular, we use Jakes' model ${ }^{2}$ for the spatial correlation with element spacing of 0.2 wavelength for $\boldsymbol{\Sigma}_{M}$, i.e., $\left(\boldsymbol{\Sigma}_{M}\right)_{i, l}=J_{0}(0.4 \cdot \pi \cdot|i-l|)$, $i, l \in\{1,2, \ldots, L\}$, where $J_{0}(\cdot)$ is the zero-order Bessel function of the first type [59].

\footnotetext{
${ }^{2}$ It is noted that Jakes' model requires the radiating patterns to share the same azimuth [59], which is a reasonable assumption for metasurface antenna elements.
} 
- $\{\boldsymbol{D}[\tau]\}_{\tau=0}^{m_{g}}$ are $U \times U$ diagonal matrices representing the attenuation coefficients, based on the model used in [2]. In particular, we set $(\boldsymbol{D}[\tau])_{i, i}=\frac{\zeta_{i}[\tau]}{\rho_{i}^{2}}$, where $\left\{\zeta_{i}[\tau]\right\}$ are the shadow fading coefficients, independently randomized from a log-normal distribution with standard deviation of $8 \mathrm{~dB}$.

Since the radiating elements in the DMA microstrips are sub-wavelength separated, the additive noise $\mathbf{w}[i]$ is inherently spatially correlated. Accounting for the coupling between the DMA elements, we set $\boldsymbol{C}_{W}=\sigma_{W}^{2} \cdot \boldsymbol{\Sigma}_{R}$, where $\boldsymbol{\Sigma}_{R}$ is the matrix representing the correlation due to sub-wavelength element spacing defined above, and $\sigma_{W}^{2}>0$ models the average power of the noise signal.

In the following we numerically evaluate the following achievable average sum-rates:

- $R_{\mathrm{s}}^{\mathrm{UC}}$ - unconstrained weights, i.e., $\mathcal{Q}=\mathcal{C}$.

- $R_{\mathrm{s}}^{\mathrm{AO}}$ - amplitude only weights, here $\mathcal{Q}=[0.001,5]$.

- $R_{\mathrm{s}}^{\mathrm{BA}}$ - binary amplitude weights, $\mathcal{Q}=\{0,0.1\}$.

- $R_{\mathrm{S}}^{\mathrm{LP}}$ - Lorentzian-constrained phase, namely, $\mathcal{Q}=$ $\left\{\frac{j+e^{j \phi}}{2}: \phi \in[0,2 \pi]\right\}$.

To compare the performance achievable with DMAs to conventional analog combining, as in, e.g., [5], [6], [10], we also compute the rate when standard analog combining architectures with $K \mathrm{RF}$ chains are used. In particular, we simulate a fully connected phase shift network (Architecture A.1 in [6]) and a fully connected switching network (Architecture A.3 in [6]), both obtained using MaGiQ algorithm [10, Sec. $\mathrm{V}-\mathrm{A}]$. The resulting achievable average sum-rates are denoted $R_{\mathrm{s}}^{\mathrm{AC}}$ and $R_{\mathrm{s}}^{\mathrm{SN}}$, respectively. Since existing works on analog combining design assume memoryless channels ${ }^{3}$, we simulate these setups only for the frequency flat scenarios in Subsection IV-A. Our results are averaged over 1000 Monte-Carlo simulations.

\section{A. Flat Channel with Identical Frequency Selectivity}

We first consider the case where the channel is frequencyflat, namely, $m_{g}=0$, and each element in the DMA exhibits the same frequency selectivity profile, as studied in Subsections III-A and III-B. In Figs. 5-6 we let the SNR, defined as $1 / \sigma_{W}^{2}$, vary in the range $[-5,30] \mathrm{dB}$. Note that here the term SNR refers only the energy of the noise, and does not account for the attenuation induced by the channel, which depends on the specific realization of the location of each UT. As the generated channels induce severe attenuation, the resulting achievable rate values are significantly smaller than those reported in previous related works, e.g., [6], [7], in which the SNR encapsulates the channel attenuation. It is also noted that in the previous works [6], [7] the rate measure represents the overall achievable rate in point-to-point MIMO communications, and not the achievable average sum-rate of a multi-user MIMO network, which can be viewed as the overall achievable rate divided by the number of UTs. For each SNR value we compare the average sum-rates achievable using DMAs with $K=10$ microstrips, each with $L$ radiating

\footnotetext{
${ }^{3}$ While it may be possible to extend analog combining design algorithm such as MaGiQ [10] to frequency selective channels, such an extension is beyond the scope of this work.
}

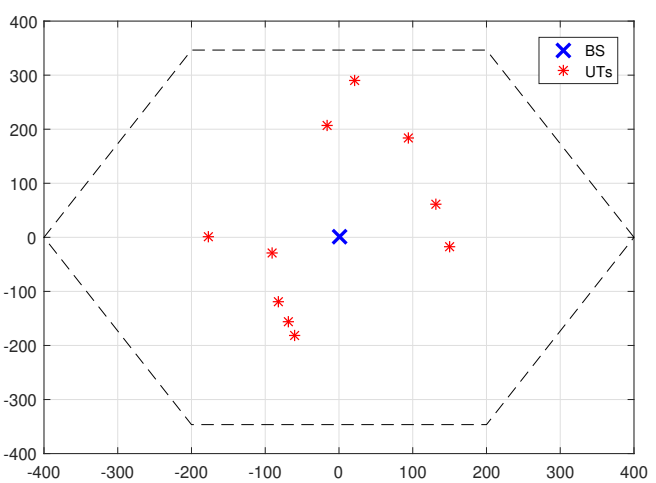

Fig. 4. Multi-user MIMO network illustration.

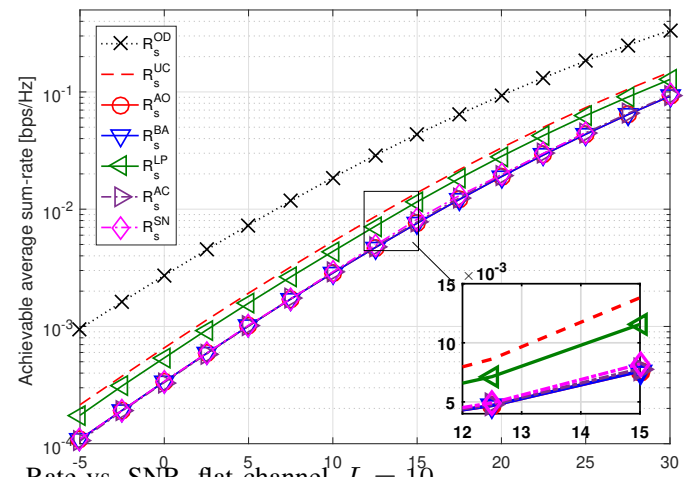

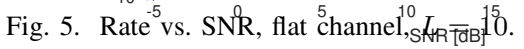

elements, computed via Algorithm 1, to the optimal achievable performance $R_{\mathrm{s}}^{\mathrm{OD}}$ computed via Corollary 2. In Fig. 5 we set $L=10$ while in Fig. 6 we use $L=15$. Recall that since $K \geq U$, then, based on the discussion following Corollary $2, R_{\mathrm{S}}^{\mathrm{OD}}$ equals the fundamental performance limit, $R_{\mathrm{s}}^{\mathrm{OM}}$, stated in Corollary 1. Observing Figs. 5-6, we note that $R_{\mathrm{s}}^{\mathrm{LP}}$ approaches $R_{\mathrm{S}}^{\mathrm{UC}}$ for all SNR values when $L=10$ and for SNR above $15 \mathrm{~dB}$ when $L=15$. This indicates that the Lorentzian-constrained phase restriction induces negligible loss when designing the weights using Algorithm 1. The amplitude only restriction, the binary amplitude constraint, the standard phase shifting network, and the standard switching network, all achieve roughly the same performance, which is within a small gap of that achievable using the Lorentzianconstrained phase weights. Furthermore, the SNR loss induced by restricting the weights matrix to satisfy (3), namely, the fact that the DMA combines only inputs from the same microstrip, is approximately $7 \mathrm{~dB}$. In particular, for $L=10, R_{\mathrm{s}}^{\mathrm{OD}}$, which is achieved without the structure constraint (3), achieves an average sum-rate of $0.1 \mathrm{bps} / \mathrm{Hz}$ at SNR of $17 \mathrm{~dB}$, while $R_{\mathrm{s}}^{\mathrm{UC}}$ achieves the same performance for SNR of $24 \mathrm{~dB}$. For $L=15$, $R_{\mathrm{s}}^{\mathrm{OD}}=0.1$ for $\mathrm{SNR}$ of $20 \mathrm{~dB}$, while $R_{\mathrm{s}}^{\mathrm{UC}}$ achieves this sum-rate at SNR of $27 \mathrm{~dB}$. Furthermore, it is noted that both curves scale similarly with respect to SNR, indicating that any average sum-rate which is achievable using an optimal unconstrained antenna array, is also achievable using practical DMA setups as the SNR increases.

Next, in Fig. 7, we fix the SNR to $15 \mathrm{~dB}$, the number of antennas to $N=90$, and compute the achievable average 
sum-rates for $K \in[1,18]$. The goal of this study is to numerically evaluate how the number of microstrips effects the performance for a given number of antennas. In order to guarantee that the same channel and noise statistics are used for each value of $K$, we fix the coupling matrix $\Sigma_{R}$ to $\boldsymbol{\Sigma}_{R}=\boldsymbol{I}_{15} \otimes \boldsymbol{\Sigma}_{M}$, where $\boldsymbol{\Sigma}_{M}$ is a $6 \times 6$ matrix defined earlier in this section, representing the element coupling via Jakes' model. Observing Fig. 7, we again note that the performance achievable with practical Lornetzian-constrained phase weights approaches that achieved with unconstrained weights for most considered values of $K$, where the gap between $R_{\mathrm{s}}^{\mathrm{LP}}$ and the unconstrained $R_{\mathrm{s}}^{\mathrm{UC}}$ is at most $1.3 \cdot 10^{-2} \mathrm{bps} / \mathrm{Hz}$. As expected, for $K=1, R_{\mathrm{s}}^{\mathrm{UC}}$, which is subject only to (3), coincides with the optimal performance $R_{\mathrm{s}}^{\mathrm{OD}}$, as (3) imposes no constraint on the weights matrix structure for $K=1$. The performance gap of the DMA-based receivers from the optimal $R_{\mathrm{S}}^{\mathrm{OD}}$ depends on the number of micropstrips $K$. For example, for $K=6$, we observe that $R_{\mathrm{s}}^{\mathrm{LP}}=1.5 \cdot 10^{-2} \mathrm{bps} / \mathrm{Hz}$, while $R_{\mathrm{s}}^{\mathrm{AO}}$ and $R_{\mathrm{s}}^{\mathrm{BA}}$ are approximately $1 \cdot 10^{-2} \mathrm{bps} / \mathrm{Hz}$, i.e., gaps of roughly $4 \cdot 10^{-2} \mathrm{bps} / \mathrm{Hz}$ and $4.5 \cdot 10^{-2} \mathrm{bps} / \mathrm{Hz}$, respectively, from the optimal performance $R_{\mathrm{s}}^{\mathrm{OD}}=5.5 \cdot 10^{-2}$. This gap becomes less dominant as $K$ further increases, and for $K=15$ it is reduced to approximately $3.5 \cdot 10^{-2} \mathrm{bps} / \mathrm{Hz}$ for all considered DMA-based receivers. Additionally, we note that $R_{\mathrm{S}}^{\mathrm{OD}}$ is monotonically increasing for small values of $K$, and for $K>3$ its value remains constant and equals the fundamental limit of the channel, $R_{\mathrm{s}}^{\mathrm{OM}}$. This follows since, as noted in the discussion following Corollary $2, R_{\mathrm{s}}^{\mathrm{OD}}$ remains constant when $K$ is larger than the rank of $\tilde{\boldsymbol{G}}$. Our numerical study shows that for the considered scenario, most realizations of $\tilde{\boldsymbol{G}}$ have at most 3 dominant eigenvalues. This behavior is due to the fact that the diagonal entries of the attenuation coefficients matrix $D$, which is randomized using the statistical model of [2], exhibit notable variations, as UTs located at different distances from the BS can observe substantially different attenuation coefficients. For this reason, $R_{\mathrm{s}}^{\mathrm{OD}}$ remains constant for $K>3$. Since the constraint induced on $R_{\mathrm{S}}^{\mathrm{UC}}$ in (3) becomes less significant as $K$ decreases, and since, as noted in Fig. $5, R_{\mathrm{s}}^{\mathrm{LP}}$ is capable of approaching $R_{\mathrm{s}}^{\mathrm{UC}}$ at such SNRs, it is shown in Fig. 7 that, for a fixed number of elements $N$, both $R_{\mathrm{s}}^{\mathrm{UC}}$ and $R_{\mathrm{s}}^{\mathrm{LP}}$ do not necessarily increase when the number of microstrips $K$ is increased.

While the results in Fig. 7 may be in favor of setting $K=1$ and $L=N$, in practice increasing the number of elements on a single microstrip increases the attenuation which results from the propagation of the signal inside the microstrip. This phenomena is not accounted for in the model here, which assumes that the attenuation induced by each element is identical, hence the additional loss by increasing the number of elements per microstrip is not reflected in Fig. 7. This observation also implies that in a practical implementation, we need to strike a balance between the cost (proportional to the number of RF ports or $K$ ), losses (proportional to the number of metamaterial elements, $L$ ), and the performance (related to both quantities). This investigation is left for future works.

We also note that restricting the weights to binary values achieves roughly the same performance as continuous

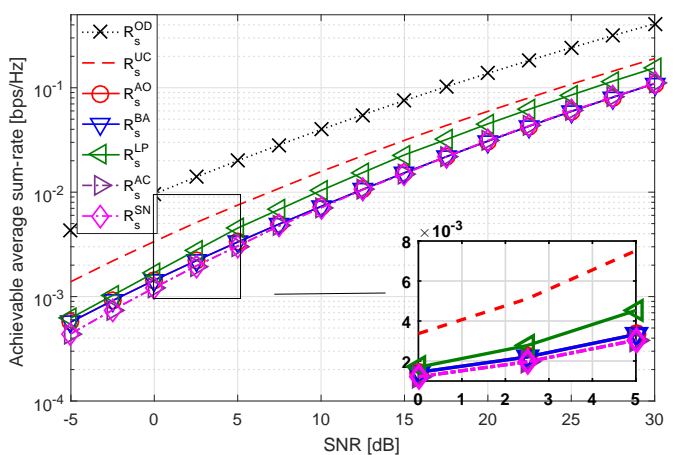

Fig. 6. Rate vs. SNR, flat channel, $L=15$.

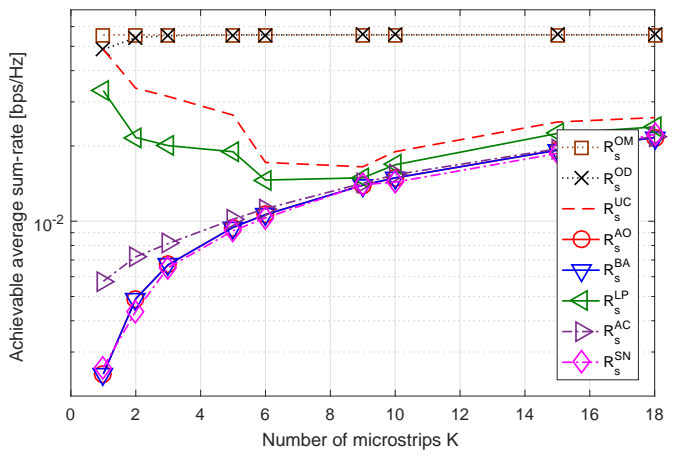

Fig. 7. Rate vs. microstrips, flat channel.

valued amplitude weights, and that under both constraints, the achievable average sum-rate substantially increases as $K$ increases where the gap from the optimal MIMO $R_{\mathrm{s}}$ varies from approximately $6 \cdot 10^{-2} \mathrm{bps} / \mathrm{Hz}$ for $K=1$ to $3.5 \cdot 10^{-2}$ bps/Hz for $K=18$. The standard analog combining networks $R_{\mathrm{s}}^{\mathrm{AC}}$ and $R_{\mathrm{s}}^{\mathrm{SN}}$, which also depend on $K$ as its value here determines the number of RF chains, achieve approximately the same performance as DMAs with continuous valued amplitude weights, where the phase shifters network achieves a slightly better performance for $K<9$.

Finally, we observe in Fig. 7 that, while $R_{\mathrm{s}}^{\mathrm{OD}}$ remains constant as the number of microstrips $K$ increases above $U$, the performance achievable with DMAs is monotonically increasing. This follows since, as discussed in Subsection III-B, increasing the number of microstrips $K$ allows designing the matrices $\tilde{\boldsymbol{U}}$ and $\tilde{\boldsymbol{D}}$ in (13), which have no effect on the resulting optimal performance $R_{\mathrm{s}}^{\mathrm{OD}}$, such that the resulting $Q^{\mathrm{OD}}$ can be better approximated using a feasible weights matrix.

The results presented in this subsection demonstrate that for frequency flat channels, BSs equipped with DMAs can achieve a performance which is comparable with costly optimal unconstrained antenna arrays. Furthermore, by utilizing our proposed alternating optimization algorithm, the sum-rate achievable with DMAs is not smaller and even larger than that achieved using standard fully connected analog combiners, obtained using state-of-the-art design algorithms.

\section{B. Varying Frequency Selectivity}

Next, we consider the more general setup where the channel is frequency selective and each element exhibits a different fre- 


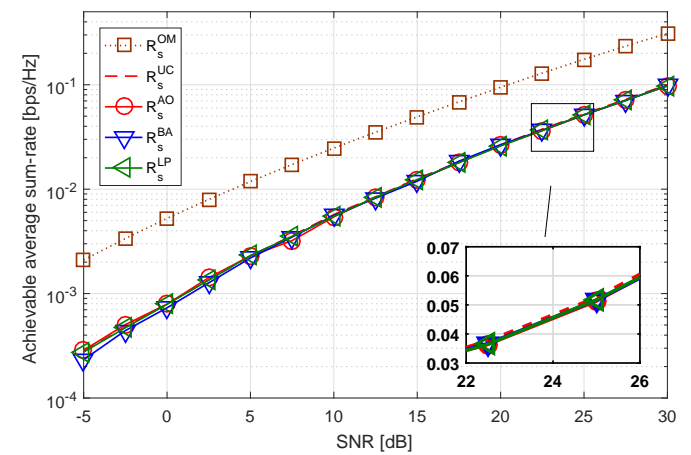

Fig. 8. Rate vs. SNR, frequency selective channel.

quency selectivity profile. In particular, we consider a channel with two taps, i.e., $m_{g}=1$, and set $\boldsymbol{\Gamma}(\omega)=\boldsymbol{I}_{K} \otimes \boldsymbol{\Gamma}_{G}(\omega)$, where $\boldsymbol{\Gamma}_{G}(\omega) \in \mathcal{C}^{L \times L}$ is a diagonal matrix representing the frequency selectivity profile of single microstrip. Based on the model detailed in Subsection II-A, we account for the frequency response of the elements and the propagation inside the waveguide via the setting $\left(\boldsymbol{\Gamma}_{G}(\omega)\right)_{l, l}=e^{-(\alpha+j \cdot \beta(\omega)) \cdot l}$. In particular, we set $\beta(\omega)=1.592 \cdot \omega\left[\mathrm{m}^{-1}\right]$ and $\alpha=0.0006$ $\left[\mathrm{m}^{-1}\right]$, representing a microstrip with $50 \mathrm{ohm}$ characteristic impedance made of Duroid 5880 operating at $1.9 \mathrm{GHz}$ with element spacing of 0.2 wavelength (assuming free space wavelength) [60, Ch. 3.8].

Fig. 8 depicts the average sum-rates achievable using DMAs configured via Algorithm 2 versus SNR, for $K=10$ and $L=$ 10 . The performance is compared to the theoretical limit $R_{\mathrm{s}}^{\mathrm{OM}}$. Observing Fig. 8 we note that unlike the scenario considered in the previous subsection, here the performance achievable with DMAs for all considered feasible sets $\mathcal{Q}$ is roughly the same. We also note that the achievable performance with DMA is within a notable gap of approximately $10 \mathrm{~dB}$ in SNR from the upper bound on the maximal achievable performance in Proposition 1. The increased gap stems from the fact that, as shown in the proof of Proposition $1, R_{\mathrm{s}}^{\mathrm{OM}}$ is obtained by mitigating the frequency selectivity of the wireless channel and the metamaterial elements. Unlike the scenario considered in Subsection IV-A whose results are depicted in Fig. 5, here the frequency selectivity induced by the physics of the metasurface cannot be mitigated by properly setting the coefficients matrix $Q$, and thus the difference between upper bound $R_{\mathrm{s}}^{\mathrm{OM}}$ and the achievable performance with DMAs increases. Despite this gap, it is observed in Fig. 8 that the performance achievable with DMAs scales similarly to the upper bound $R_{\mathrm{s}}^{\mathrm{OM}}$ with respect to SNR, indicating that the performance achievable with DMAs is comparable with $R_{\mathrm{s}}^{\mathrm{OM}}$.

Finally, in Fig. 9 we depict the achievable sum-rate versus number of microstrips for fixed number of antennas $N=90$ and SNR of $15 \mathrm{~dB}$. These rates are compared to the theoretical limit $R_{\mathrm{s}}^{\mathrm{OM}}$, as well as to the upper bound on DMA performance computed via Proposition 1, which coincides with the theoretical limit $R_{\mathrm{s}}^{\mathrm{OM}}$ for $K \geq U$. Observing Fig. 9 we note that here, unlike the results depicted Fig. 7 which considered a similar scenario but did not account for the signal propagation inside the microsrtip, increasing the number of microstrips

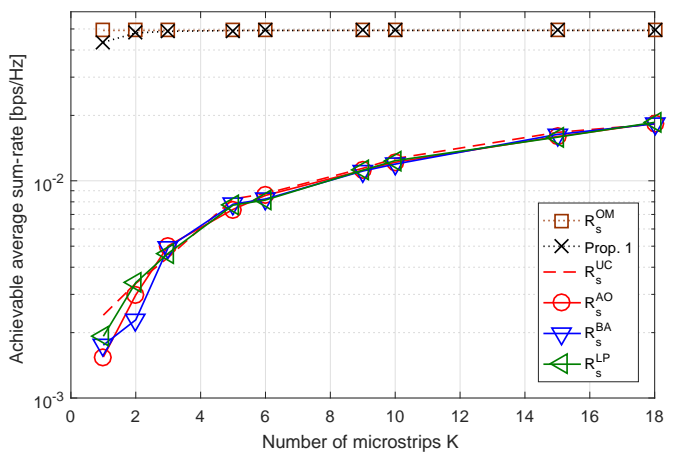

Fig. 9. Rate vs. microstrips, frequency selective channel.

improves the performance. This follows since increasing the number of elements in each microsrtip induces additional attenuation, which impairs the ability of the BS to recover the messages from the channel output. It is also observed in Fig. 9 that the performance achievable using DMAs is roughly the same for all settings of $\mathcal{Q}$, as observed in Fig. 8 . The fact that the binary amplitude setting, which is relatively simple to implement, achieves roughly the same performance as the other settings, makes it an appealing candidate for future practical implementations studies. Lastly, we note that there is a notable gap between the upper bound of Proposition 1, which is computed by letting the DMA weights be frequency selective, and the actual sum-rate achievable with fixed weights. This indicates that the performance achievable with DMAs can be substantially improved in frequency selective channels by designing the frequency response of each element, which is modeled in the DMA weights, to vary in frequency as in, e.g., [61]. We leave the analysis and design of such DMAs for future work.

\section{CONCLUSions}

In this work we studied massive MIMO systems where the large-scale antenna array at the BS is implemented using a DMA. We characterized the maximal achievable average sum-rate on the uplink, and derived two alternating optimization algorithms for designing practical DMAs to approach the optimal performance: the first algorithm is designed for frequency flat channels assuming that the frequency selectivity induced by the metasurface is identical among all elements, and the second algorithm generalizes the first algorithm to arbitrary multipath channels and frequency selectivity profiles. Our results illustrate the potential gains over standard antenna arrays of utilizing DMAs for implementing compact low-cost and low-power massive MIMO systems. In particular, it is shown in our simulations study that by properly adjusting the inherent combining and compression induced by the physics of DMAs, a practical massive MIMO system can be constructed which is capable of achieving performance comparable to the fundamental theoretical limits, which require costly, power consuming, and large-sized optimal antenna arrays.

\section{A. Proof of Theorem 1}

\section{APPENDIX}

To prove the theorem, we first write the operation of the DMA as part of the massive MIMO channel. By combining (4) 
and (2), and defining $\tilde{\mathbf{w}}[i] \triangleq \boldsymbol{Q} \sum_{\tau=0}^{m_{h}} \boldsymbol{H}[\tau] \mathbf{w}[i-\tau]$ and $\tilde{\boldsymbol{H}}[\tau] \triangleq$ $\boldsymbol{Q} \sum_{l=0}^{m_{g}} \boldsymbol{H}[\tau-l] \boldsymbol{G}[l]$, the equivalent input-output relationship can be written as

$$
\mathbf{z}[i]=\sum_{\tau=0}^{m_{h}+m_{g}} \tilde{\boldsymbol{H}}[\tau] \mathbf{x}[i-\tau]+\tilde{\mathbf{w}}[i] .
$$

Note that $\tilde{\mathbf{w}}[i]$ in (A.1) is a stationary multivariate propercomplex Gaussian process with finite memory $m_{h}+m_{g}$. Consequently, (A.1) represents a finite-memory Gaussian MAC. Thus, by letting $\boldsymbol{S}_{\mathbf{x}}(\omega), \boldsymbol{S}_{\tilde{\mathbf{w}}}(\omega)$, and $\tilde{\boldsymbol{\Gamma}}(\omega), \omega \in[0,2 \pi)$, denote the power spectral density (PSD) of $\mathbf{x}[i]$, PSD of $\tilde{\mathbf{w}}[i]$, and the DTFT of $\tilde{\boldsymbol{H}}[\tau]$, respectively, it follows that the achievable average sum-rate is given by [41]

$$
\begin{aligned}
& R_{\mathrm{s}}=\frac{1}{U} \lim _{l \rightarrow \infty} \frac{1}{l} I\left(\mathbf{x}^{l} ; \mathbf{z}^{l}\right) \\
&= \frac{1}{2 \pi \cdot U} \int_{0}^{2 \pi} \log \left|\boldsymbol{I}_{K}+\tilde{\boldsymbol{\Gamma}}(\omega) \boldsymbol{S}_{\mathbf{x}}(\omega) \tilde{\boldsymbol{\Gamma}}^{H}(\omega)\left(\boldsymbol{S}_{\tilde{\mathbf{w}}}(\omega)\right)^{-1}\right| d \omega \\
& \stackrel{(a)}{=} \frac{1}{2 \pi \cdot U} \int_{0}^{2 \pi} \log \mid \boldsymbol{I}_{K}+\boldsymbol{Q} \boldsymbol{\Gamma}(\omega) \boldsymbol{\Sigma}(\omega) \boldsymbol{\Sigma}^{H}(\omega) \boldsymbol{\Gamma}^{H}(\omega) \boldsymbol{Q}^{H} \\
& \quad \times\left(\boldsymbol{Q} \boldsymbol{\Gamma}(\omega) \boldsymbol{C}_{W} \boldsymbol{\Gamma}^{H}(\omega) \boldsymbol{Q}^{H}\right)^{-1} \mid d \omega, \quad \text { (A.2) }
\end{aligned}
$$

where $(a)$ follows since $\mathbf{x}[i]$ and $\mathbf{w}[i]$ are i.i.d. processes with covariances $\boldsymbol{I}_{U}$ and $\boldsymbol{C}_{W}$, respectively. Note that (A.2) coincides with (7), thus proving the theorem.

\section{B. Proof of Lemma 2}

The equality (15a) follows from the definition of the Frobenious norm, i,e,.

$$
\|\boldsymbol{Q}-\boldsymbol{M}\|^{2}=\sum_{i=1}^{K} \sum_{l=1}^{N}\left|(\boldsymbol{Q})_{i, l}-(\boldsymbol{M})_{i, l}\right|^{2} .
$$

Since the feasible set $\mathcal{Q}^{K \times N}$ is defined entry-wise, the Frobenious norm is minimized by entry-wise projection.

Similarly, the minimizing diagonal matrix in $(15 c)-(15 d)$ is obtained since

$$
\begin{aligned}
& \left\|\boldsymbol{M}_{1}-\tilde{\boldsymbol{D}} \boldsymbol{M}_{2}\right\|^{2}=\sum_{i=1}^{K}\left\|\mathbf{m}_{1, i}-(\tilde{\boldsymbol{D}})_{i, i} \cdot \mathbf{m}_{2, i}\right\|^{2} \\
& \stackrel{(a)}{=} \sum_{i=1}^{K}\left\|\mathbf{m}_{1, i}\right\|^{2}-2 \operatorname{Re}\left(\mathbf{m}_{1, i}^{H} \mathbf{m}_{2, i}\right) \cdot(\tilde{\boldsymbol{D}})_{i, i}+\left\|\mathbf{m}_{2, i}\right\|^{2} \cdot(\tilde{\boldsymbol{D}})_{i, i}^{2},
\end{aligned}
$$

where $(a)$ holds as $(\tilde{\boldsymbol{D}})_{i, i}$ is real-valued. Consequently, for each $i \in\{1,2, \ldots, K\}$, the optimal setting of $(\tilde{\boldsymbol{D}})_{i, i} \geq \epsilon$ is given by (15d).

Finally, the minimizing unitary matrix in (15b) is obtained from the unitary Procrustes problem [56, Ch. 7.4], concluding the proof of the lemma.

\section{Proof of Proposition 1}

The proposition is obtained by letting $Q$ vary with $\omega$, i.e., replacing $\boldsymbol{Q}$ in (7) with $\boldsymbol{Q}(\omega)$. Next, we define $\tilde{\boldsymbol{Q}}(\omega) \triangleq$
$\boldsymbol{Q}(\omega) \boldsymbol{\Gamma}(\omega)$. Since $\boldsymbol{\Gamma}(\omega)$ is non-singular, $\boldsymbol{Q}(\omega)$ can be recovered from $\tilde{\boldsymbol{Q}}(\omega)$. Under this setting, (7) satisfies

$$
\begin{gathered}
R_{\mathrm{s}}=\frac{1}{2 \pi} \int_{0}^{2 \pi} \frac{1}{U} \log \mid \boldsymbol{I}_{K}+\boldsymbol{Q}(\omega) \boldsymbol{\Sigma}(\omega) \boldsymbol{\Sigma}^{H}(\omega) \boldsymbol{Q}^{H}(\omega) \\
\times\left(\boldsymbol{Q}(\omega) \boldsymbol{C}_{W} \boldsymbol{Q}^{H}(\omega)\right)^{-1} \mid d \omega \\
\stackrel{(a)}{\leq} \frac{1}{2 \pi \cdot U} \int_{0}^{2 \pi} \sum_{i=1}^{\min (U, K)} \log \left(1+\lambda_{i}(\omega)\right) d \omega
\end{gathered}
$$

where $(a)$ follows from upper-bounding the integrand for each $\omega \in[0,2 \pi)$, using Corollary 2 , thus proving (19).

\section{REFERENCES}

[1] T. L. Marzetta. "Massive MIMO: An introduction". Bell Labs Technical Journal, vol. 20, Mar. 2015, pp. 11-22.

[2] T. L. Marzetta. "Noncooperative cellular wireless with unlimited numbers of base station antenna". IEEE Trans. Wireless Commun., vol. 9, no. 11 , Nov. 2010, pp. 3950-3600.

[3] J. Hoydis, S. Ten Brink, and M. Debbah. "Massive MIMO in the UL/DL of cellular networks: How many antennas do we need?". IEEE J. Sel. Areas Commun., vol. 31, no. 2, Feb. 2013, pp. 160-171.

[4] N. Shlezinger and Y. C. Eldar. "On the spectral efficiency of noncooperative uplink massive MIMO systems". IEEE Trans. Commun., vol. 67, no. 3, Mar. 2019, pp. 1956-1971.

[5] A. AlKhateeb, J. Mo, N. Gonzalez-Prelcic, and R. W. Heath. "MIMO precoding and combining solutions for millimeter-wave systems". IEEE Comm. Mag., vol. 52, no. 12, Dec. 2014, pp. 122-131.

[6] R. Mendez-Rial, C. Rusu, N. Gonzalez-Prelcic, A. AlKhateeb, and R. W. Heath. "Hybrid MIMO architectures for millimeter wave communications: Phase shifters or switches?". IEEE Access, vol. 4, 2016, pp. 247-267.

[7] J. Mo, A. Alkhateeb, S. Abu-Surra, and R. W. Heath. "Hybrid architectures with few-bit ADC receivers: Achievable rates and energyrate tradeoffs". IEEE Trans. Wireless Commun., vol. 16, no. 4, Apr. 2017, pp. 2274-2287.

[8] A. Hoeher and N. Doose. "A massive MIMO terminal concept based on small-size multi-mode antennas". Trans. Emerging Tel. Tech., vol. 28, no. 2, Feb. 2017.

[9] I. F. Akyildiz and J. M. Jornet. "Realizing Ultra-Massive MIMO (1024 $\times 1024)$ communication in the $(0.06-10)$ Terahertz band". Nano Commun. Networks, vol. 8, 2016, pp. 46-54.

[10] S. Stein and Y. C. Eldar. "Hybrid analog-digital beamforming for massive MIMO systems". IEEE Trans. Signal Process., early access, 2019.

[11] Y. Li, C. Tao, G. Seco-Granados, A. Mezghani, A. L. Swindlehurst, and L. Liu. "Channel estimation and performance analysis of one-bit massive MIMO systems". IEEE Trans. Signal Process., vol. 65, no. 15, Aug. 2017, pp. 4075-4089.

[12] J. Choi, J. Mo, and R. W. Heath. "Near maximum-likelihood detector and channel estimator for uplink multiuser massive MIMO systems with one-bit ADCs". IEEE Trans. Commun., vol. 64, no. 5, May 2016, pp. 2005-2018.

[13] J. Choi, B. L. Evans, and A. Gatherer. "Resolution-adaptive hybrid MIMO architectures for millimeter wave communications". IEEE Trans. Signal Process., vol. 65, no. 23, Dec. 2017, pp. 6201-6216.

[14] T. C. Zhang, C. K. Wen, S. Jin, and T. Jiang. "Mixed-ADC massive MIMO detectors: Performance analysis and design optimization". IEEE Trans. Wireless Commun., vol. 15, no. 11, Nov. 2016, pp. 7738-7752.

[15] J. Choi, J. Sung, B. L. Evans, and A. Gatherer. "Antenna selection for large-scale MIMO systems with low-resolution ADCs". Proc. IEEE ICASSP, Calgary, Canada, Apr. 2018.

[16] S. K. Mohammed and E. G. Larsson. "Per-antenna constant envelope precoding for large multi-user MIMO systems". IEEE Trans. Commun., vol. 61, no. 3, Mar. 2013, pp. 1059-1071.

[17] C. Studer and E. G. Larsson. "PAR-aware large-scale multi-user MIMOOFDM downlink". IEEE J. Sel. Areas Commun., vol. 31, no. 2, Feb. 2013, pp. 303-313. 
[18] S. Ghosh, T. N. Tran, and T. Le-Ngoc. "Dual-layer EBG-based miniaturized multi-element antenna for MIMO systems". IEEE Trans. Antennas Propag., vol. 62, no. 8, Aug. 2014, pp. 3985-3997.

[19] R. O. Ouedraogo, E. J. Rothwell, A. R. Diaz, K. Fuchi, and A. Temme. "Miniaturization of patch antennas using a metamaterialinspired technique". IEEE Trans. Antennas Propag., vol. 60, no. 5, May 2012, pp. 2175-2182.

[20] D. R. Smith, O. Yurduseven, L. Pulido-Mancera, P. Bowen, and N. B. Kundtz. "Analysis of a waveguide-fed metasurface antenna". Phys. Rev. Applied, vol. 8, no. 5, Nov. 2017.

[21] J. C. Mikala, S. L. Brunton, N. B. Kundtz, and N. J. Kutz. "Sidelobe canceling for reconfigurable holographic metamaterial antenna". IEEE Trans. Antennas Propag., vol. 63, no. 4, Apr. 2015, pp. 1881 - 1886.

[22] J. C. Mikala, S. L. Brunton, N. B. Kundtz, and N. J. Kutz. "Extremumseeking control of the beam pattern of a reconfigurable holographic metamaterial antenna". JOSA A., vol. 33, no. 1, Jan. 2016, pp. 59 - 68.

[23] J. G. Andrews, S. Buzzi, W. Choi, S. V. Hanly, A. Lozano, A. C. K Soong, and J. C. Zhang. "What will 5G be?". IEEE J. Sel. Areas Commun., vol. 32, no. 6, Jun. 2014, pp. 1065-1082.

[24] P. Mookiah and K. P. Dandekar. "Metamaterial-substrate antenna array for MIMO communication system". IEEE Trans. Antennas Propag., vol. 57, no. 10, Oct. 2009, pp. 3283-3292.

[25] C. Huang, A. Zappone, G. C. Alexandropoulos, M. Debbah, and C. Yuen. "Large intelligent surfaces for energy efficiency in wireless communication". arXiv preprint, arXiv:1810.06934, 2018.

[26] N. Kaina, M. Dupre, G. Lerosey, and M. Fink. "Shaping complex microwave fields in reverberating media with binary tunable metasurfaces". Nature Scientific Reports, vol. 4, no. 6693, Oct. 2014.

[27] P. del Hougne, M. Fing, and H. Lerosey. "Optimal communication channels in a disordered world with tamed randomness". arXiv preprint, arXiv:1810.00430, 2018.

[28] J. Hunt, T. Driscoll, A. Mrozack, G. Lipworth, M. Reynolds, D. Brady, and D. R. Smith. "Metamaterial apertures for computational imaging". Science, vol. 339, no. 6117, Jan. 2013, pp. 310-313.

[29] T. Sleasman, M. F. Imani, W. Xu, J. Hunt, T. Driscoll, M. S. Reynolds, and D. R. Smith. "Waveguide-fed tunable metamaterial element for dynamic apertures". IEEE Antennas Wirel Propag Lett., vol. 15, 2016, pp. $606-609$.

[30] A. V. Diebold, M. F. Imani, T. Sleasman, and D. R. Smith. "Phaseless computational ghost imaging at microwave frequencies using a dynamic metasurface aperture". Applied Optics, vol. 57, no. 9, 2018, pp. 2142 2149.

[31] T. Sleasman, M. Boyarsky, M. F. Imani, J. N. Gollub, and D. R. Smith. "Design considerations for a dynamic metamaterial aperture for computational imaging at microwave frequencies". J. Opt. Soc. Am. B, vol. 33, no. 6, 2016, pp. 1098 - 1111.

[32] T. Sleasman, M. Boyarsky, M. F. Imani, T. Fromenteze, J. N. Gollub, and D. R. Smith. "Single-frequency microwave imaging with dynamic metasurface apertures". J. Opt. Soc. Am. B, vol. 34, no. 8, 2017, pp. $1713-1736$.

[33] T. Sleasman, M. Boyarsky, L. Pulido-Mancera, T. Fromenteze, M. F. Imani, M. S. Reynolds, and D. R. Smith. "Experimental synthetic aperture radar with dynamic metasurfaces". IEEE Trans. Antennas Propag., vol. 65, no. 12, Dec. 2017, pp. 6864 - 6877.

[34] M. Boyarsky, T. Sleasman, L. Pulido-Mancera, T. Fromenteze, A Pedross-Engel, C. M. Watts, M. F. Imani, M. S. Reynolds, and D. R. Smith. "Synthetic aperture radar with dynamic metasurface antennas: a conceptual development". J. Opt. Soc. Am. A, vol. 34, no. 5, 2017, pp. A22 - A36.

[35] C. M. Watts, A. Pedross-Engel, D. R. Smith, and M. S. Reynolds. "Xband SAR imaging with a liquid-crystal-based dynamic metasurface antenna". J. Opt. Soc. Am. B, vol. 34, no.28, 2017, pp. 300 - 306.

[36] M. C. Johnson, S. L. Brunton, N. B. Kundtz, and J. N. Kutz. "Sidelobe canceling for reconfigurable holographic metamaterial antenna". IEEE Trans. Antennas Propag., vol. 63, no. 4, Apr. 2015, pp. 1881-1886.

[37] I. Yoo, M. F. Imani, T. Sleasman, H. D. Pfister, and D. R. Smith. "Enhancing capacity of spatial multiplexing systems using reconfigurable cavity-backed metasurface antennas in clustered MIMO channels". IEEE Trans. Commun., vol. 67, no. 2, Feb. 2019, pp. 1070-1084.

[38] J. B. Pendry, A. J. Holden, D. J. Robbins, and W. J. Stewart. "Magnetism from conductors and enhanced nonlinear phenomena". IEEE Trans. Microw. Theory Tech., vol. 47, no. 11, Nov. 1999, pp. 2075-2084.

[39] L. M. Pulido-Mancera, T. Zvolensky, M. F. Imani, P. T. Bowen, M. Valayil, and D. R. Smith. "Discrete dipole approximation applied to highly directive slotted waveguide antennas". IEEE Antennas Wirel Propag Lett., vol. 15, 2016, pp. 1823 - 1826.
[40] L. M. Pulido-Mancera, M. F. Imani, P. T. Bowen, N. Kundtz, and D. R. Smith. "Analytical modeling of a two-dimensional waveguide-fed metasurface". arXiv preprint, arXiv:1807.11592, 2018.

[41] S. Verdu. "Multiple-access channels with memory with and without frame synchronism," IEEE Trans. on Inform. Theory, vol. 35, no. 3 May 1989, pp. 605-619.

[42] N. Engheta and R. W. Ziolkowski. Metamaterials: Physics and Engineering Explorations. John Wiley \& Sons, 2006

[43] D. R. Smith, J. B. Pendry, and M. C. K. Wiltshire. "Metamaterials and negative refractive index". Science, vol. 305, no. 5685, Aug. 2004, pp. 788-792.

[44] C. L. Holloway, E. F. Kuester, J. A. Gordon, J. O'Hara, J. Booth, and D. R. Smith. "An overview of the theory and applications of metasurfaces: The two-dimensional equivalents of metamaterials". IEEE Antennas Propag. Mag., vol. 54, no. 2, Apr. 2012, pp. 10 - 35.

[45] C. Pfeiffer and A. Grbic. "Metamaterial Huygens surfaces: tailoring wave fronts with reflectionless sheets". Physical Review Letters, vol. 110, no. 19, May 2013, pp. 197401.

[46] H. Odabasi, F. L. Teixeira, and D. O. Guney. "Electrically small, complementary electric-field-coupled resonator antennas". Journal of Applied Physics, vol. 113, no. 8, 2013, pp. 084903.

[47] V. Kristem, C. U. Bas, R. Wang, and A. F. Molisch. "Outdoor wideband channel measurements and modeling in the $3-18 \mathrm{GHz}$ band". IEEE Trans. Wireless Commun., vol. 17, no. 7, Jul. 2018, pp. 4620-4633.

[48] D. N. C. Tse and P. Viswanath. Fundamentals of Wireless Communication. Cambridge, 2005.

[49] N. Shlezinger, Y. C. Eldar, and M. R. D. Rodrigues. "Hardware-limited task-based quantization". arXiv preprint, arXiv:1807.08305, 2018.

[50] L. H. Brandenburg and A. D. Wyner. "Capacity of the Gaussian channel with memory: The multivariate case," Bell System Technical Journal, vol. 53, no. 5, May. 1974, pp. 745-778.

[51] A. Goldsmith and M. Effros. "The capacity region of broadcast channels with intersymbol interference and colored Gaussian noise," IEEE Trans. Inform. Theory, vol. 47, no. 1, pp. 219-240, Jan. 2001.

[52] N. Shlezinger, D. Zahavi, Y. Murin, and R. Dabora. "The secrecy capacity of Gaussian MIMO channels with finite memory," IEEE Trans. Inform. Theory, vol. 63, no. 13, Mar. 2017, pp. 1874-1897.

[53] A. El Gamal and Y. H. Kim. Network Information Theory. Cambridge, 2011

[54] C. D. Meyer. Matrix Analysis and Applied Linear Algebra. Society for Industrial and Applied Mathematics, 2000

[55] N. Shlezinger, R. Dabora, and Y. C. Eldar. "Measurement matrix design for phase retrieval based on mutual information". IEEE Trans. Signal Process., vol. 66, no. 2, Jan. 2018, pp. 324-339.

[56] R. A. Horn and C. A. Johnson. Matrix Analysis. Cambridge, 1990.

[57] J. C. Bezdek and R. J. Hathaway. "Convergence of alternating optimization". Neural, Parallel and Scientific Computations, vol. 11, no. 4, Dec. 2003, pp. 351-368.

[58] C. Xiao, J. Wu, S. Y. Leong, Y. R. Zheng, and K. Ben Letaief. "A discrete-time model for triply selective MIMO Rayleigh fading channels". IEEE Trans. Wireless Commun., vol. 3, no. 5, Sep. 2004 pp. $1678-1688$.

[59] W. C. Jakes. Microwave Mobile Communications. IEEE Press, 1993.

[60] D. M. Pozar. Microwave Engineering. Wiley Press, 2009.

[61] I. Yoo, M. F. Imani, T. Sleasman, and D. R. Smith. "Efficient complementary metamaterial element for waveguide-fed metasurface antennas". Optics Express, vol. 24, no. 25, 2016, pp. 28686 - 28692. 